JOURNAL OF

SYMPLECTIC GEOMETRY

Volume 2, Number 1, 133 - 175, 2003

\title{
MOMENT MAPS AND EQUIVARIANT SZEGÖ KERNELS
}

\section{RoBerto PaOLETTI}

Let $M$ be a connected $n$-dimensional complex projective manifold and consider an Hermitian ample holomorphic line bundle $\left(L, h_{L}\right)$ on $M$. Suppose that the unique compatible covariant derivative $\nabla_{L}$ on $L$ has curvature $-2 \pi i \Omega$, where $\Omega$ is a Kähler form. Let $G$ be a compact connected Lie group and $\mu: G \times M \rightarrow M$ a holomorphic Hamiltonian action on $(M, \Omega)$. Let $\mathfrak{g}$ be the Lie algebra of $G$, and denote by $\Phi: M \rightarrow \mathfrak{g}^{*}$ the moment map.

Let us also assume that the action of $G$ on $M$ linearizes to a holomorphic action on $L$; given that the action is Hamiltonian, the obstruction for this is of topological nature [GS1]. We may then also assume that the Hermitian structure $h_{L}$ of $L$, and consequently the connection as well, are $G$-invariant. Therefore for every $k \in \mathbb{N}$ there is an induced linear representation of $G$ on the space $H^{0}\left(M, L^{\otimes k}\right)$ of global holomorphic sections of $L^{\otimes k}$. This representation is unitary with respect to the natural Hermitian structure of $H^{0}\left(M, L^{\otimes k}\right)$ (associated to $\Omega$ and $h_{L}$ in the standard manner). We may thus decompose $H^{0}\left(M, L^{\otimes k}\right)$ equivariantly according to the irreducible representations of $G$.

The subject of this paper is the local and global asymptotic behaviour of certain linear series defined in terms this decomposition. Namely, we shall first consider the asymptotic behaviour as $k \rightarrow+\infty$ of the linear subseries of $H^{0}\left(M, L^{\otimes k}\right)$ associated to a single irreducible representation, and then of the linear subseries associated to a whole ladder of irreducible representations. To this end, we shall estimate the asymptoptic growth, in an appropriate local sense, of these linear series on some loci in $M$ defined in terms of the moment map $\Phi$.

To express the problem in point more precisely, fix a Cartan subalgebra $\mathfrak{h} \subset \mathfrak{g}$, and let $H \subset G$ be the corresponding Cartan subgroup. Let $R \subset \mathfrak{h}^{*}$ be the root system for $(\mathfrak{g}, \mathfrak{h})$, and fix a basis $S=\left\{\alpha_{i}\right\} \subset R$ for $R$. Let $X(H)$ be the group of characters of $H$, identified with a subgroup of $\mathfrak{h}^{*}$ (a character $\chi: H \rightarrow \mathbb{C}^{*}$ is determined by its differential at the identity). The finite dimensional irreducible representations of $G$ correspond bijectively to points in $X(H) \cap \mathfrak{h}_{+}^{*}$, where $\mathfrak{h}_{+}^{*} \subset \mathfrak{h}^{*}$ is the positive Weyl chamber [S]. Let $E_{\omega}$ be the irreducible representation associated to $\omega \in X(H) \cap \mathfrak{h}_{+}^{*}$. We have 
for every $k \geq 0$ a $G$-equivariant orthogonal decomposition

$$
H^{0}\left(M, L^{\otimes k}\right) \cong \bigoplus_{\omega \in X(H) \cap \mathfrak{h}_{+}^{*}} H^{0}\left(M, L^{\otimes k}\right)_{\omega},
$$

where each $H^{0}\left(M, L^{\otimes k}\right)_{\omega}$ is equivariantly isomorphic to a direct sum of copies of $E_{\omega}$. We shall first focus on the asymptotic behaviour, as $k \rightarrow+\infty$, of the linear series $\left|H^{0}\left(M, L^{\otimes k}\right)_{\omega}\right|$ for a fixed $\omega \in X(H) \cap \mathfrak{h}_{+}^{*}$, under certain transversality assumptions on the moment map $\Phi$.

Let us now clarify what is meant here by local asymptotic behaviour of a family of linear series such as $\left|H^{0}\left(M, L^{\otimes k}\right)_{\omega}\right|$. Suppose first that $V \subseteq$ $H^{0}(M, L)$ is any nonzero vector subspace, and let $\left\{s_{j}\right\}$ be an orthonormal basis of $V$ for the induced unitary structure. Consider the function

$$
\nu_{V}(p)=\sum_{j}\left\|s_{j}(p)\right\|_{p}^{2} \quad(p \in M)
$$

where \|\|$_{p}$ denotes the Hermitian norm of $L$ at $p$. This is in fact independent of the choice of orthonormal basis, as one can see by replacing the $s_{j}$ 's by $t_{k}=\sum_{j} u_{j k} s_{j}$ where $U=\left[u_{i j}\right] \in U(\operatorname{dim}(V))$ is a unitary matrix, and then working in a local frame. By the same reason, given that the representation of $G$ on $H^{0}(M, L)$ is unitary, if $V \subseteq H^{0}(M, L)$ is $G$-invariant then so is the function $\nu_{V}$. Here we shall be interested in sequences of functions such as $\nu_{H^{0}\left(M, L^{\otimes k}\right)_{\omega}}$ and in their asymptotic behaviour.

Pictorially, we may think of $\nu_{V}(p)$ as measuring the local size, under the chosen metric structures, of $V$ at $p$. Asymptotic expansions such as (3) below have attracted interest in algebraic geometry since Zelditch remarked that they control the metric (equivalently, symplectic) asymptotic behaviour of the maps to projective space associated to the linear series at hand $[\mathbf{Z}]$.

Theorem 1. Suppose that $0 \in \mathfrak{g}^{*}$ is a regular value of the moment map $\Phi: M \rightarrow \mathfrak{g}^{*}$ and that $G$ acts freely on $\Phi^{-1}(0)$. Set $n=\operatorname{dim}_{\mathbb{C}}(M), g=$ $\operatorname{dim}_{\mathbb{R}}(G)$. There exist on $\Phi^{-1}(0)$ smooth $G$-invariant real valued functions $a_{0}>0$ and, for every $\omega \in X(H) \cap \mathfrak{h}_{+}^{*}$ and $l \geq 1$, $a_{l}^{(\omega)}$ with the following property: Suppose given $\omega \in X(H) \cap \mathfrak{h}_{+}^{*}$ and the assignment, for every $k \in \mathbb{N}$, of an orthonormal basis $\left\{s_{j}^{(k, \omega)}\right\}$ of $H^{0}\left(M, L^{\otimes k}\right)_{\omega}$. Then there is for $k \gg 0$ an asymptotic development, uniform in $p \in \Phi^{-1}(0)$,

$$
\begin{aligned}
\sum_{j}\left\|s_{j}^{(k, \omega)}(p)\right\|^{2} \sim & k^{n-g / 2} \operatorname{dim}\left(V_{\omega}\right)^{2} a_{0}(p) \\
& +\sum_{l \geq 1} k^{n-l-g / 2} a_{l}^{(\omega)}(p) .
\end{aligned}
$$

Here $V_{\omega}$ denotes the irreducible representation associated to $\omega$. 
If $\tilde{G}$ denotes the complexification of $G$, the actions of $G$ on $M$ and $L$ extend to holomorphic actions of $\tilde{G}$. Given any subset $A \subseteq M$, we shall denote by $\tilde{G} \cdot A$ its saturation under $\tilde{G}$, that is,

$$
\tilde{G} \cdot A=\{g \cdot m: g \in \tilde{G}, m \in M\} .
$$

In the hypothesis of the Theorem, $\tilde{G}$ acts freely on $\tilde{G} \cdot \Phi^{-1}(0)$, and the latter is the open subset of stable points for the action of $G$ on $M$ [GS1].

Corollary 1. Under the hypothesis of Theorem 1, there exists $k_{\omega}$ such that for $k \geq k_{\omega}$ the base locus of the linear series $\left|H^{0}\left(M, L^{\otimes k}\right)_{\omega}\right|$ satisfies

$$
\operatorname{Bs}\left(\left|H^{0}\left(M, L^{\otimes k}\right)_{\omega}\right|\right) \subseteq M \backslash\left(\tilde{G} \cdot \Phi^{-1}(0)\right) .
$$

Away from $\Phi^{-1}(0), \sum_{j}\left\|s_{j}^{(k, \omega)}(p)\right\|^{2}$ is rapidly decreasing in $k$, uniformly so on the complement of the unstable locus. More precisely, let $R=M \backslash$ $\left(\tilde{G} \cdot \Phi^{-1}(0)\right)$ be the set of unstable points of the action. Then,

Proposition 1. In the situation of Theorem 1 , if $p \notin \Phi^{-1}(0)$ then

$$
\sum_{j}\left\|s_{j}^{(k, \omega)}(p)\right\|^{2}=O\left(k^{-N}\right), \quad N=1,2, \ldots
$$

This estimate is uniform on compact subsets of $M \backslash\left(\Phi^{-1}(0) \cup R\right)$.

Let us at least briefly describe how Theorem 1 generalizes when the action of $G$ on $\Phi^{-1}(0)$ is not free. Since 0 is a regular value, the action of $G$ on $\Phi^{-1}(0)$ is at any rate locally free, and therefore the stabilizer subgroup $G_{p}$ of any $p \in \Phi^{-1}(0)$ is finite. Furthermore, there is an induced unitary action $\alpha_{p}: G_{p} \rightarrow S^{1}$ on the Hermitian complex line $\left(L_{p}, h_{p}\right)$. We may then take the $L^{2}$-product of $\alpha_{p}$ and $\chi_{\omega}$ on $G_{p}$ with respect to the counting measure, that is,

$$
\left(\chi_{\omega}, \alpha_{p}\right)_{G_{p}}=\sum_{g \in G_{p}} \chi_{\omega}(g) \cdot \overline{\alpha_{p}(g)} .
$$

Then (3) is replaced by

$$
\sum_{j}\left\|s_{j}^{(k, \omega)}(p)\right\|^{2} \sim k^{n-g / 2} \operatorname{dim}\left(V_{\omega}\right)\left(\chi_{\omega}, \alpha_{p}^{k}\right)_{G_{p}} a_{0}(p)+\sum_{l \geq 1} k^{n-l-g / 2} a_{l}^{(\omega)}(p),
$$

uniformly in $p \in \Phi^{-1}(0)$.

The microlocal techniques used in the proof of Theorem 1 can also be used to study the asymptotic growth of the dimension of the spaces of equivariant sections $H^{0}\left(M, L^{\otimes k}\right)_{\omega}$ when $\omega$ is kept fixed and $k \rightarrow+\infty$. There already exist two approaches to this problem, one geometric and the other algebraic. 
A geometric solution follows from Meinrenken's proof of a fundamental conjecture of Guillemin and Sternberg $[\mathbf{M}]$, resting on the symplectic cutting technique of E. Lerman [L]: Obviously $G$ acts on every cohomology group $H^{i}(M, L), i=0, \ldots, n$, and we may consider the virtual vector space

$$
\operatorname{RR}(M, L)_{\omega}=\sum_{i=0}^{n}(-1)^{i} H^{i}(M, L)_{\omega} .
$$

Let $\mu_{\omega}=\mu_{\omega}(M, L)$ be the corresponding virtual multiplicity for the representation $V_{\omega}$. By the main Theorem of $[\mathbf{M}], \mu_{\omega}$ may be computed as a Riemann-Roch number on the symplectic reduction $M_{\omega}$ of the Hamiltonian $G$-manifold $(M, \Omega, \Phi)$ at $\omega$, provided $\omega$ is a regular value of the moment map. Then $M_{\omega}=\Phi^{-1}(\omega) / G_{\omega}$, where $G_{\omega} \subseteq G$ is the stabilizer subgroup of $\omega$ under the coadjoint action. Assuming to fix ideas that $G_{f}$ acts freely on $\Phi^{-1}(\omega), M_{\omega}$ naturally inherits by restriction and quotient a Kähler structure and a compatible polarization $L_{\omega}$. Then by Theorem 1.1 and Corollary 1.2 of $[\mathbf{M}]$

$$
\mu_{\omega}=\operatorname{RR}\left(M_{\omega}, L_{\omega}\right)
$$

Let us now replace $L$ by $L^{\otimes k}$, and thus $\Omega$ by $k \Omega$ and $\Phi$ by $\Phi_{k}=k \Phi$. By Serre vanishing if $k \gg 0$ then $H^{i}\left(M, L^{\otimes k}\right)=0$ for all $i>0$, and therefore $\operatorname{RR}\left(M, L^{\otimes k}\right)_{\omega}=H^{0}\left(M, L^{\otimes k}\right)_{\omega}$ and $\mu_{\omega}\left(L^{\otimes k}\right)$ is the multiplicity of $V_{\omega}$ in $H^{0}\left(M, L^{\otimes k}\right)$. Thus,

$$
\operatorname{dim} H^{0}\left(M, L^{\otimes k}\right)_{\omega}=\operatorname{dim}\left(V_{\omega}\right) \cdot \operatorname{RR}\left(M_{\omega, k}, L_{\omega, k}^{\otimes k}\right),
$$

where $M_{\omega, k}=\Phi^{-1}\left(k^{-1} \omega\right) / G_{\omega}$ is the reduction of $\left(M, k \Omega, \Phi_{k}\right)$ at $\omega$ and $L_{\omega, k}^{\otimes k}$ is the polarization on it induced by $L^{\otimes k}$. Now by Corollary 7.3 of $[\mathbf{M}]$ the reduced spaces $M_{\omega, k}$ are all diffeomorphic to the fibration

$$
M\left(P, \mathcal{O}_{\omega}\right)=P \times{ }_{G} \mathcal{O}_{\omega},
$$

where $P=\Phi^{-1}(0)$ is viewed as a principal $G$-bundle over the symplectic quotient $M_{0}=\Phi^{-1}(0) / G$, and $\mathcal{O}_{\omega}$ is the coadjoint orbit of $\omega$. On the other hand, again by Corollary 7.3 of [M], the symplectic structure $\Omega_{\omega, k}$ on $M_{\omega, k}$ is the one induced by minimal coupling [GS3] from $k \Omega$, the symplectic structure $\sigma_{k^{-1} \omega}$ on $\mathcal{O}_{k^{-1} \omega}$, a fixed connection on $P$ and the moment map $J_{\omega, k}=\frac{1}{k} J_{\omega}: \mathcal{O}_{\omega} \hookrightarrow \mathfrak{g}^{*}$ of $\mathcal{O}_{\omega, k}$. With the appropriate scaling taken into account, one obtains a leading asymptotics of degree $n-g$.

There is also an algebraic line of research on this asymptotic problem, in the work of Brion and Dixmier [BD], $[\mathbf{B}]$.

Here we propose a different, analytic and fairly elementary approach to the same asymptotics: namely, we use Boutet de Monvel and Sjöstrand's microlocal description of the Szegö kernel $[\mathbf{B S}]$ to reduce the problem to an application of the stationary phase Lemma along $\Phi^{-1}(0)$. Behind some technicalities, the basic idea is very simple. 
In this formulation, the asymptotics depends on the weight $\omega$ only through the germ at the identity $e \in G$ of its charachter function $\chi_{\omega} \in \mathcal{C}^{\infty}(G)$, rather than on the geometry of the coadjoint orbit $\mathcal{O}_{\omega}$.

Theorem 2. In the hypothesis and notation of Theorem 1, there exist for $j \geq 1$ differential polynomials $S_{j}$ of degree $2 j$ on a neighbourhood of $e \in G$ such that for any $\omega \in X(H) \cap \mathfrak{h}_{+}^{*}$ we have

$$
\begin{aligned}
\operatorname{dim} H^{0}\left(M, L^{\otimes k}\right)_{\omega} \sim & \operatorname{dim}\left(V_{\omega}\right)^{2} \cdot \operatorname{vol}\left(M_{0}\right) \cdot k^{n-g} \\
& +\operatorname{dim}\left(V_{\omega}\right) \sum_{j \geq 1} b_{j, \omega} k^{n-g-j},
\end{aligned}
$$

where $\operatorname{vol}\left(M_{0}\right)$ is the volume of the reduced space $M_{0}=: \Phi^{-1}(0) / G$ with its natural Kähler structure, and $b_{j, \omega}=S_{j}\left(\chi_{\omega}\right)(e)$.

It follows from the proof of Theorem 2 (and the stationary phase Lemma) that the $S_{j}$ 's may be expressed in terms of the Hessian of $h_{L}$ along $\Phi^{-1}(0)$ and the classical symbol appearing in the Fourier integral representation of the Szegö kernel of $\Pi$ given in [BS].

This extends to our setting a (special case of a) result of Brion and Dixmier $[\mathbf{B D}],[\mathbf{B}]$ :

Corollary 2. In the hypothesis of Theorem 1 , let $\mu_{\omega, k}$ be the multiplicity of $V_{\omega}$ in $H^{0}\left(M, L^{\otimes k}\right)_{\omega}$. Then

$$
\lim _{k \rightarrow+\infty} \frac{\mu_{\omega, k}}{\mu_{0, k}}=\operatorname{dim}\left(V_{\omega}\right) .
$$

When $0 \notin \Phi(M)$, a more informative result is provided by the study of the corresponding asymptotic properties of the ladder linear series

$$
H^{0}\left(M, L^{\otimes k}\right)_{(\omega)}=: \bigoplus_{\ell=1}^{\infty} H^{0}\left(M, L^{\otimes k}\right)_{\ell \omega} \subseteq H^{0}\left(M, L^{\otimes k}\right) .
$$

We shall now assume that $G$ is semisimple.

The action of $G$ on $L$ induces an action on the dual line bundle $L^{*}$, equipped with the dual hermitian metric. The unit circle bundle $X \subseteq L^{*}$ is invariant under $G$, and therefore there is an induced Hamiltonian action of $G$ on the cotangent bundle $T^{*} X$, and on the complement of the zero section $T^{*} X \backslash\{0\}$. Let $\Psi: T^{*} X \backslash\{0\} \rightarrow \mathfrak{g}^{*}$ be the corresponding (conic) moment map.

Since the connection is $G$-invariant, the action on $T^{*} X$ preserves the positive cone $Y \subseteq T^{*} X$ generated by the normalized connection 1-form $\alpha$ :

$$
Y=\left\{\left(x, r \alpha_{x}\right): x \in X, r>0\right\} .
$$

It is well-known that since $\Omega$ is symplectic $Y$ is in fact a symplectic conic submanifold of $T^{*} X$. 
The moment maps $\Psi$ and $\Phi$ are tightly related on $Y$ : one has

$$
\Psi\left(\left(x, r \alpha_{x}\right)\right)=r \Phi(\pi(x)), \quad(x \in X)
$$

where $\pi: X \rightarrow M$ is the projection [GS1].

We need a further piece of notation [GS2].

Definition 1. For $\omega \in X(H) \cap \mathfrak{h}_{+}^{*}$, we shall denote by $\mathbb{R}_{+} \omega=\{r \cdot \omega: r>0\}$ the positive ray through $\omega$. Let furthermore $\mathcal{O}=\mathcal{O}_{\omega} \subseteq \mathfrak{g}^{*}$ be the coadjoint orbit of $\omega$. We shall denote by $C(\mathcal{O}) \subseteq \mathfrak{g}^{*}$ the positive cone over $\mathcal{O}$, that is,

$$
C(\mathcal{O})=\{r \lambda: r>0, \lambda \in \mathcal{O}\} .
$$

Notice that $\Phi: M \rightarrow \mathfrak{g}^{*}$ is transversal to $C(\mathcal{O})$ if and only if so is $\left.\Psi\right|_{Y}$. Since $\left.\Psi\right|_{Y}$ is conic, the latter condition is in turn equivalent to the one that $\left.\Psi\right|_{Y}$ be transversal to $\mathcal{O}$, and by $G$-equivariance this is in turn equivalent to the condition that $\omega$ be a regular value of $\left.\Psi\right|_{Y}$. Hence, in view of the invariance of the connection 1-form, conditions ii) and iii) below are equivalent to the one that $\omega$ should lie in an elementary fundamental wedge for the induced Hamiltonian action of $G$ on $Y$, in the terminology of [GS2], page 357.

Theorem 3. Suppose $G$ is semisimple, compact and connected. Given $\omega \in$ $X(H) \cap \mathfrak{h}_{+}^{*}$, suppose that

i): $0 \notin \Phi(M)$;

ii): $\Phi$ is transversal to $C(\mathcal{O})$;

iii): the stabilizer subgroup $G_{\omega} \subseteq G$ of $\omega$ acts freely on $\Phi^{-1}\left(\mathbb{R}_{+} \omega\right)$ (equivalently, $G_{\omega}$ acts freely on $\left.\Phi^{-1}(C(\mathcal{O}))\right)$.

Then there exist smooth $G$-invariant functions

$$
b_{l}: \Phi^{-1}(C(\mathcal{O})) \rightarrow \mathbb{R} \quad(l=0,1, \ldots)
$$

with $b_{0}>0$ such that for every choice of orthonormal basis $\left\{s_{j}^{(k, \ell \omega)}\right\}$ of $H^{0}\left(M, L^{\otimes k}\right)_{(\ell \omega)}$ for $\ell=1,2, \ldots$, there is an asymptotic development

$$
\sum_{\ell, j}\left\|s_{j}^{(k, \ell \omega)}(p)\right\|^{2} \sim \sum_{l \geq 0} k^{n-l} b_{l}(p),
$$

uniformly in $p \in \Phi^{-1}(C(\mathcal{O}))$. Furthermore,

$$
\sum_{\ell, j}\left\|s_{j}^{(k, \ell \omega)}(p)\right\|^{2}=O\left(k^{-N}\right), \quad N=1,2, \ldots,
$$

uniformly on compact subsets of $M \backslash \Phi^{-1}(C(\mathcal{O}))$. 
Corollary 3. Under the hypothesis of Theorem 3, there exists $k_{\omega}$ such that for $k \geq k_{\omega}$ the base locus of the linear series $\left|H^{0}\left(M, L^{\otimes k}\right)_{(\omega)}\right|$ satisfies

$$
\operatorname{Bs}\left(\left|H^{0}\left(M, L^{\otimes k}\right)_{(\omega)}\right|\right) \subseteq M \backslash(\tilde{G} \cdot W),
$$

where $W=\Phi^{-1}(C(\mathcal{O}))$.

By restriction of the arguments in the proof of Theorem we have:

Corollary 4. If the hypothesis of Theorem 3 are satisfied on a $G$-invariant open set $M^{\prime}=M \backslash B$, where $B \subseteq M$ has measure zero, then the same conclusions hold for $p \in M^{\prime}$.

Example 1. If $\Omega_{F S}$ is the Fubini-Study form on $\mathbb{P}^{1}$, let $\Omega=: 2 \pi_{1}^{*}\left(\Omega_{F S}\right)+$ $\pi_{2}^{*}\left(\Omega_{F S}\right)$, where $\pi_{i}: \mathbb{P}^{1} \times \mathbb{P}^{1} \rightarrow \mathbb{P}^{1}$ is the projection on the $i$-th factor $(i=$ $1,2)$. Consider the diagonal action of $\mathrm{SU}(2)$ on $\left(\mathbb{P}^{1} \times \mathbb{P}^{1}, \Omega\right)$. The moment map $\hat{\Phi}: \mathbb{P}^{1} \times \mathbb{P}^{1} \rightarrow \mathfrak{s u}(2)^{*}$ for this action is $\hat{\Phi}(p, q)=2 \Phi(p)+\Phi(q)$, where $\Phi: \mathbb{P}^{1} \rightarrow \mathfrak{s u}(2)^{*}$ is the moment map for the action on $\left(\mathbb{P}^{1}, \Omega_{F S}\right)$. We may equivariantly identify $\mathbb{P}^{1}$ with the unit sphere $S^{2}$ and $\mathfrak{s u}(2)^{*} \cong \mathfrak{s u}(2) \cong \mathbb{R}^{3}$, so that $\Phi$ corresponds to the inclusion $\iota: S^{2} \hookrightarrow \mathbb{R}^{3}$. Then the hypothesis in Theorem 3 and its Corollary 4 are satisfied on the complement of the diagonal, $M^{\prime}=\left(\mathbb{P}^{1} \times \mathbb{P}^{1}\right) \backslash \Delta_{\mathbb{P}^{1}}$ (strictly speaking, after replacing the action of $\mathrm{SU}(2)$ on $\mathbb{P}^{1}$ by the action of $\mathrm{SO}(3)$ on $\left.S^{2}\right)$. If $H$ denotes the hyperplane line bundle on $\mathbb{P}^{1}$, the line bundle on $\mathbb{P}^{1} \times \mathbb{P}^{1}$ associated to $\Omega$ is $L=H^{\otimes 2} \otimes H$. Set $V=\mathbb{C}^{2}$. For every $k \geq 1$,

$$
H^{0}\left(\mathbb{P}^{1} \times \mathbb{P}^{1}, L^{\otimes k}\right) \cong \operatorname{Sym}^{2 k}\left(V^{*}\right) \otimes \operatorname{Sym}^{k}\left(V^{*}\right) \cong \bigoplus_{0 \leq j \leq k} \operatorname{Sym}^{3 k-2 j}\left(V^{*}\right) .
$$

For any integer $r \geq 1$, let $H^{0}\left(\mathbb{P}^{1} \times \mathbb{P}^{1}, L^{\otimes k}\right)_{(r)} \subseteq H^{0}\left(\mathbb{P}^{1} \times \mathbb{P}^{1}, L^{\otimes k}\right)$ be the subspace corresponding to the direct sum of the terms $\operatorname{Sym}^{3 k-2 j}\left(V^{*}\right)$ with $3 k-2 j \equiv 0(\bmod r)$. By the Theorem and its Corollary, for any fixed $(p, q) \in\left(\mathbb{P}^{1} \times \mathbb{P}^{1}\right) \backslash \Delta_{\mathbb{P}^{1}}$ and any fixed integer $r \geq 1$ the asymptotic growth as $k \rightarrow+\infty$ of the local size at $(p, q)$ of the linear series $H^{0}\left(\mathbb{P}^{1} \times \mathbb{P}^{1}, L^{\otimes k}\right)_{(r)}$ grows like $a_{r}(p, q) k^{2}$ for some $a_{r}(p, q)>0$.

These results are based on the microlocal description of the Szegö kernel given by Boutet de Monvel and Sjöstrand in [BS]. In particular, Theorem 3 is proved by giving a similar microlocal description of the orthogonal projector associated to the linear series $H^{0}\left(M, L^{\otimes k}\right)_{(\ell \omega)}$. To this end, we shall also rely on the microlocal description of a projector associated to ladders of representations given by Guillemin and Sternberg in [GS2], and on a reduction technique used by Schiffman and Zelditch in $[\mathbf{S Z}]$. Overall, as the reader will easily see, the paper is also largely in debt to arguments from [GS1], [SZ] and $[\mathbf{Z}]$. 
In future work we shall consider extensions of these results to the almost complex setting, and further investigate the asymptotic growth of the spaces of equivariant sections.

Acknowledgments. I am endebted to the referee for some fruitful and stimulating comments.

\section{Proof of Theorem 1}

Let $L^{*}$ be the dual line bundle of $L$, with the induced Hermitian metric and connection, and let

$$
\rho: L^{*} \rightarrow \mathbb{R}, \quad(x, v) \mapsto\|v\|_{x}^{2},
$$

be the associated square norm function. Let $X \subset L^{*}$ be the unit circle bundle:

$$
X=\{(p, v): \rho((p, v))=1\},
$$

with projection $\pi: X \rightarrow M$. We shall denote by $p, q, \ldots$ points in $M$, and by $x, y, \ldots$ points in $X$.

By the ampleness of $L, X$ is the boundary of the bounded strictly pseudoconvex domain $D=\{\rho \leq 1\}$. If $i \alpha$ is the connection form on $X$, then $\alpha$ is a contact form, $d \alpha=\pi^{*}(\Omega)$ and $\alpha \wedge \pi^{*}(\Omega)^{\wedge n}$ is a volume form on $X$. Given this, we shall implicitly identify functions and half-forms.

There is a canonical isomorphism for every $k$ between the spaces of smooth sections of $L^{\otimes k}$ on $M, \mathcal{C}^{\infty}\left(M, L^{\otimes k}\right)$, and the spaces $\mathcal{C}^{\infty}(X)_{k}$ of smooth functions on $X$ of the $k$-th isotype for the $S^{1}$-action. We shall occasionally denote by $\tilde{V} \subseteq \mathcal{C}^{\infty}(X)_{k}$ the subspace corresponding to a subspace $V \subseteq$ $\mathcal{C}^{\infty}\left(M, L^{\otimes k}\right)$, and occasionally not distinguish between the two.

Let $\Pi \in \mathcal{D}^{\prime}(X \times X)$ be the Szegö kernel, that is, the distributional kernel of the orthogonal projector $\pi: L^{2}(X) \rightarrow H(X)$, where $H(X)$ is the Hardy

space of boundary values on $X$ of holomorphic functions on $D$. If $\left\{s_{j}^{(k)}\right\}_{j=1}^{N_{k}}$ is an orthonormal basis of $H^{0}\left(M, L^{\otimes k}\right)$ for every $k \geq 0$, viewed implicitly as a space of CR functions on $X$, we have

$$
\Pi(x, y)=\sum_{k=0}^{+\infty} \Pi_{k}(x, y) \quad(x, y \in X)
$$

where

$$
\Pi_{k}(x, y)=\sum_{j=1}^{N_{k}} s_{j}^{(k)}(x) \otimes \bar{s}_{j}^{(k)}(y) \quad(x, y \in X, k \geq 0) .
$$


As proved in $[\mathbf{B S}], \Pi$ is a Fourier integral with complex phase. More precisely, it is microlocally equivalent to an oscillatory integral of the form

$$
\Pi(x, y)=\int_{0}^{+\infty} e^{i t \psi(x, y)} s(x, y, t) d t \quad(x, y \in X),
$$

where $s \in S^{n}\left(X \times X \times \mathbb{R}_{+}\right)$has an asymptotic expansion

$$
s(x, y, t) \sim \sum_{j=0}^{\infty} s_{j}(x, y) t^{n-j}
$$

The restriction to the diagonal of the principal term $s_{0}(x, x)$ is given explicitly in equation (4.10) of [BS]:

$$
s_{0}(x, x)=\frac{1}{4 \pi^{n}} \operatorname{det}\left(L_{X}(x)\right) \cdot\|d \rho\| \quad(x \in X),
$$

where $L_{X}$ is the Levi form. We refer to $[\mathbf{B S}],[\mathbf{Z}]$, [SZ] for a discussion of the phase $\psi \in \mathcal{C}^{\infty}\left(L^{*} \times L^{*}\right)$; it parametrizes an almost holomorphic Lagrangian submanifold, whose real locus is the wave front of $\Pi$. This is the isotropic conic submanifold

$$
\Sigma=\left\{\left(x, r \alpha_{x}, x,-r \alpha_{x}\right): x \in X, r>0\right\} \subseteq T^{*}(X \times X) .
$$

The Taylor series of $\psi$ along the diagonal $\Delta_{L^{*}} \subset L^{*} \times L^{*}$ is completely determined (equivalently, $\psi$ is uniquely determined up to a function vanishing to infinite order along $\Delta_{L^{*}}$ ). Explicitly, if $x \in L^{*}$, in local holomorphic coordinates induced by a local holomorphic frame for $L$ in a neighbourhood of $\pi(x)$, we have

$$
\psi(x+h, x+k) \sim \frac{1}{i} \sum_{I, J} \frac{\partial^{I+J} \rho}{\partial z^{I} \partial \bar{z}^{J}}(x) \frac{h^{I}}{I !} \frac{\bar{k}^{J}}{J !} \quad\left(h, k \in \mathbb{C}^{n+1}\right) .
$$

We can retrieve $\Pi_{k}$ in (8) as the $k$-th Fourier component of $\Pi$ :

$$
\Pi_{k}(x, y)=\int_{0}^{+\infty} \int_{S^{1}} e^{-i k \theta} e^{i t \psi\left(r_{\theta} x, y\right)} s\left(r_{\theta} x, y, t\right) d t d \theta \quad(x, y \in X),
$$

where $r:\left(e^{i \theta}, x\right) \in S^{1} \times X \mapsto r_{\theta}(x) \in X$ is the $S^{1}$-action on $X$; application of the stationary phase lemma gives an asymptotic expansion for $\Pi_{k}$ in terms of which many classical results in algebraic geometry can be deduced $[\mathbf{Z}]$.

Given the direct sum decomposition (1), we may take as an orthonormal basis for $H^{0}\left(M, L^{\otimes k}\right)$ the union of a collection of orthonormal basis $\left\{s_{j}^{(k, \omega)}\right\}_{j=1}^{N_{k, \omega}}$ of $H^{0}\left(M, L^{\otimes k}\right)_{\omega}$ for each $\omega \in X(H) \cap \mathfrak{h}_{+}^{*}$. Thus,

$$
\Pi_{k}(x, y)=\sum_{\omega \in X(H) \cap \mathfrak{h}_{+}^{*}} \Pi_{k, \omega}(x, y) \quad(x, y \in X, k \geq 0),
$$


where

$$
\Pi_{k, \omega}(x, y)=\sum_{j=1}^{N_{k, \omega}} s_{j}^{(k, \omega)}(x) \otimes \bar{s}_{j}^{(k, \omega)}(y) \quad\left(k \geq 0, \omega \in X(H) \cap \mathfrak{h}_{+}^{*}\right) .
$$

If $x \in X$ and $p=\pi(x)$, we have

$$
\Pi_{k, \omega}(x, x)=\sum_{j=1}^{N_{k, \omega}}\left\|s_{j}^{(k, \omega)}(p)\right\|^{2} .
$$

Thus we want to study the asymptotic behaviour of $\Pi_{k, \omega}(x, x)$ for a fixed $\omega$ and $x \in \pi^{-1}\left(\Phi^{-1}(0)\right)$ as $k \rightarrow+\infty$.

Clearly, $\Pi_{k, \omega}$ is the $k$-th Fourier component of the equivariant Szegö kernel $\Pi_{\omega} \in \mathcal{D}^{\prime}(X \times X)$ associated to $\omega$, that is, the distributional kernel of the orthogonal projector

$$
\pi_{\omega}: L^{2}(X) \longrightarrow H(X)_{\omega}
$$

where we have set

$$
H(X)_{\omega}=\bigoplus_{k \in \mathbb{Z}} H^{0}\left(\widetilde{M, L^{\otimes k}}\right)_{\omega} .
$$

Thus, $\Pi_{\omega, k} \in \mathcal{C}^{\infty}(X \times X)$ is the distributional kernel for the orthogonal projector

$$
\left.\pi_{\omega, k}: L^{2}(X) \longrightarrow H^{0} \widetilde{\left(M, L^{\otimes k}\right.}\right)_{\omega} .
$$

Let $p_{\omega}: L^{2}(X) \rightarrow L^{2}(X)_{\omega}$ be the orthogonal projector onto the $G$ equivariant Hilbert subspace of $L^{2}(X)$ associated to $\omega$, let $q_{k}: L^{2}(X) \rightarrow$ $L^{2}(X)_{k}$ be the orthogonal projector onto the $k$-th isotype for the $S^{1}$-action, and let $\pi: L^{2}(X) \rightarrow H(X)$ be as above the orthogonal projector onto the Hardy space of CR functions. Then

$$
\pi_{\omega, k}=p_{\omega} \circ q_{k} \circ \pi
$$

Let $G$ be a compact topological group and $\sigma: G \rightarrow U(V)$ be a finite dimensional irreducible representation. Let $\varrho: G \rightarrow U(W)$ be a unitary action on a separable Hilbert space, and let $W_{V} \subseteq W$ be the equivariant $G$-suspace associated to $\sigma$. Then the orthogonal projection operator $\Pi_{V}$ : $W \rightarrow W_{V}$ is given by

$$
\Pi_{V}=\operatorname{dim}(V) \cdot \int_{G} \varrho(g) \chi_{\sigma}\left(g^{-1}\right) d g,
$$

where $\chi_{\sigma}$ is the character of $\sigma$ and $d g$ a normalized Haar measure on $G[\mathbf{D}]$.

To simplify notation, let us denote by $\tilde{\mu}$ both the linearization to $L$ of the action of $G$ on $M$, and the induced actions of $G$ on $L^{*}$ and $X$. Under the usual identifications, the linear representations of $G$ on the spaces 
$H^{0}\left(M, L^{\otimes k}\right)$ are then given by pull-back of CR functions on $X$ under this action. Thus,

$$
\begin{array}{r}
\Pi_{k, \omega}(x, y)=\operatorname{dim}\left(V_{\omega}\right) \cdot \int_{G} \chi_{\omega}\left(g^{-1}\right) \varrho(g)\left(\Pi_{k}(x, y)\right) d g \\
=\operatorname{dim}\left(V_{\omega}\right) \cdot \int_{G} \chi_{\omega}\left(g^{-1}\right) \Pi_{k}\left(\tilde{\mu}_{g^{-1}}(x), y\right) d g \\
=\operatorname{dim}\left(V_{\omega}\right) \cdot \int_{0}^{+\infty} \int_{S^{1}} \int_{G} e^{-i k \theta} e^{i t \psi\left(\tilde{\mu}_{g^{-1}} \circ r_{\theta}(x), y\right)} \cdot \chi_{\omega}\left(g^{-1}\right) \cdot \\
\cdot s\left(\tilde{\mu}_{g^{-1}} \circ r_{\theta}(x), y, t\right) d t d \theta d g \quad(x, y \in X)
\end{array}
$$

By assumption, $G$ acts freely on $\Phi^{-1}(0)$. We may thus find an open neighbourhood $U$ of the unit $e \in G$ and $\epsilon>0$ such that $d(p, g p)>\epsilon$ if $p \in \Phi^{-1}(0)$, $g \notin U$. If $x=y \in \pi^{-1}\left(\Phi^{-1}(0)\right)$, the integral (15) may thus be decomposed as follows:

$$
\begin{array}{r}
\Pi_{k, \omega}(x, x)=\operatorname{dim}\left(V_{\omega}\right) \cdot \int_{U} \chi_{\sigma}\left(g^{-1}\right) \Pi_{k}\left(\tilde{\mu}_{g^{-1}}(x), x\right) d g \\
\quad+\operatorname{dim}\left(V_{\omega}\right) \cdot \int_{G \backslash U} \chi_{\sigma}\left(g^{-1}\right) \Pi_{k}\left(\tilde{\mu}_{g^{-1}}(x), x\right) d g,
\end{array}
$$

the latter term being $O\left(k^{-N}\right)$ for every $N=1,2, \ldots$ as $k \rightarrow+\infty$, uniformly in $x \in \pi^{-1}\left(\Phi^{-1}(0)\right)$. Let us focus on the former term, which we call $\Pi_{k, \omega}(x, x)^{\prime}$. Setting $t=k u$, this may be rewritten

$$
\begin{aligned}
\Pi_{k, \omega}(x, x)^{\prime}= & k \operatorname{dim}\left(V_{\omega}\right) \cdot \int_{0}^{+\infty} \int_{S^{1}} \int_{U} e^{i k\left[u \psi\left(\tilde{\mu}_{g^{-1}} \circ r_{\theta}(x), x\right)-\theta\right]} \\
& \cdot \chi_{\sigma}\left(g^{-1}\right) \cdots s\left(\tilde{\mu}_{g^{-1}} \circ r_{\theta}(x), x, k u\right) d u d \theta d g \\
= & k \operatorname{dim}\left(V_{\omega}\right) \cdot \int_{0}^{+\infty} \int_{S^{1}} \int_{U} e^{i k \Psi(x, u, \theta, g)} \cdot \chi_{\sigma}\left(g^{-1}\right) \cdot \\
& \cdot s\left(\tilde{\mu}_{g^{-1}} \circ r_{\theta}(x), x, k u\right) d u d \theta d g .
\end{aligned}
$$

From the corresponding property of $\psi$, it follows that the phase

$$
\Psi(x, u, \theta, g)=u \psi\left(\tilde{\mu}_{g^{-1}} \circ r_{\theta}(x), x\right)-\theta
$$

has positive imaginary part. Therefore, (17) is a complex oscillatory integral, and its asymptotic behaviour as $k \rightarrow+\infty$ is determined by the stationary points of the phase as a function of $(g, \theta, t)$.

Lemma 1. Suppose $x \in \Phi^{-1}(0)$. Then $(e, 0,1)$ is a non-degenerate critical point of $\Psi$. Furthermore, perhaps after replacing $U$ with a smaller open neighbourhood of $e \in G$, it is the only critical point of $\Psi$ in $U \times S^{1} \times(0,+\infty)$.

Here, of course, we implicitly identify $\theta$ with $e^{i \theta}$. 
Proof of Lemma 1. Let us first show that $(e, 0,1)$ is a critical point of $\Psi$. We have

$$
\tilde{\mu}_{e} \circ r_{0}(x)=x \text { and }(d \psi)_{(x, x)}=\left(x, \alpha_{x}, x,-\alpha_{x}\right) .
$$

The connection on $L$ induces a $G \times S^{1}$-invariant direct sum decomposition $T X=H(X / M) \oplus V(X / M)$ into a horizontal and a vertical subbundle; here

$$
H(X / M)=\operatorname{ker}(\alpha) \text { and } V(X / M)=\operatorname{span}\left\{\frac{\partial}{\partial \theta}\right\}
$$

$\left(\frac{\partial}{\partial \theta}\right.$ denotes the generator of the $S^{1}$-action on $X$ ). If $\xi \in \mathfrak{g}$, let $\xi_{M}$ and $\xi_{X}$ denote, respectively, the vector fields induced by $\xi$ on $M$ and $X$. If $V$ is a vector field on $M$, let $V^{\sharp}$ denote its horizontal lift to $X$. Then, in terms of the above direct sum decomposition of $T X$, we have for all $\xi \in \mathfrak{g}$ [GS1]:

$$
\xi_{X}=\left(\xi_{M}^{\sharp},\left(\phi_{\xi} \circ \pi\right) \cdot \frac{\partial}{\partial \theta}\right)
$$

where $\phi_{\xi}=:\langle\Phi, \xi\rangle: M \rightarrow \mathbb{R}$ is the $\xi$-component of the moment map. In particular,

$$
\xi_{X}(x)=\xi_{M}^{\sharp}(x) \text { for every } \xi \in \mathfrak{g} \text { if } \Phi(\pi(x))=0 .
$$

Let us introduce the map

$$
a_{\xi}: \mathbb{R} \rightarrow X \times X, \quad t \mapsto\left(\tilde{\mu}_{\exp (t \xi)}(x), x\right) .
$$

Given (18) and (20),

$(21)\left(\partial_{\xi} \Psi\right)_{(e, 1,0)}=\left.\frac{d}{d t}\right|_{t=0} \psi\left(a_{\xi}(-t) x, x\right)=(d \psi)_{(x, x)}\left(-\xi_{X}(x), 0\right)=0$,

for every $\xi \in \mathfrak{g}$. On the other hand, it follows from the arguments on pages $327-328$ of $[\mathbf{Z}]$ that $(1,0)$ is the only critical point of $\Psi(x, e, \theta, u)$ as a function of $(\theta, u)$; more precisely, we have

$$
\left(d_{u} \Psi\right)_{(x, e, \theta, u)}=-i\left(1-e^{i \theta}\right) \text { and }\left(d_{\theta} \Psi\right)_{(x, e, \theta, u)}=t e^{i \theta}-1 .
$$

Furthermore $(1,0)$ is a nondegenerate critical point of $\Psi(x, e, \theta, u)$ as a function of $(\theta, u)$, and the Hessian is given there by

$$
\left[\begin{array}{ll}
i & 1 \\
1 & 0
\end{array}\right]
$$

We can now prove that $(e, 0,1)$ is a nondegenerate critical point of $\Psi$. Perhaps after restricting to a smaller open neighbourhood of $e \in G$, we may suppose that $U$ is diffeomorphic to an open neighbourhood of $0 \in \mathfrak{g}$ under the exponential map; having fixed a basis of $\mathfrak{g}$, let $\left(h_{1}, \ldots, h_{g}\right)(g=\operatorname{dim}(G))$ be the resulting coordinates on $U$ centered at $e$. By the above and the expression of $\Psi$, it is clear that $\left.\frac{\partial^{2} \Psi}{\partial h_{j} \partial u}\right|_{(e, 1,0)}=0$. Given this and (23), the 
Hessian of $\Psi(x, g, \theta, u)$ in the coordinates $\left(h_{j}, \theta, u\right)$ at $(e, 0,1)$ has the form

$$
H(\Psi)=\left[\begin{array}{ccccc}
\left.\frac{\partial^{2} \Psi}{\partial h_{1}^{2}}\right|_{(e, 0,1)} & \cdots & \left.\frac{\partial^{2} \Psi}{\partial h_{1} \partial h_{g}}\right|_{(e, 0,1)} & \left.\frac{\partial^{2} \Psi}{\partial h_{1} \partial \theta}\right|_{(e, 0,1)} & 0 \\
\vdots & \ddots & \vdots & \ddots & \vdots \\
\left.\frac{\partial^{2} \Psi}{\partial h_{g} \partial h_{1}}\right|_{(e, 0,1)} & \cdots & \left.\frac{\partial^{2} \Psi}{\partial h_{g}^{2}}\right|_{(e, 0,1)} & \left.\frac{\partial^{2} \Psi}{\partial h_{g} \partial \theta}\right|_{(e, 0,1)} & 0 \\
\left.\frac{\partial^{2} \Psi}{\partial h_{1} \partial \theta}\right|_{(e, 0,1)} & \cdots & \left.\frac{\partial^{2} \Psi}{\partial h_{g} \partial \theta}\right|_{(e, 0,1)} & i & 1 \\
0 & \cdots & 0 & 1 & 0
\end{array}\right] .
$$

Therefore,

$$
\begin{aligned}
\operatorname{det}(H(\Psi))=\operatorname{det} & \left(\left[\begin{array}{ccc}
\left.\frac{\partial^{2} \Psi}{\partial h_{1}^{2}}\right|_{(e, 0,1)} & \cdots & \left.\frac{\partial^{2} \Psi}{\partial h_{1} \partial h_{g}}\right|_{(e, 0,1)} \\
\vdots & \ddots & \vdots \\
\left.\frac{\partial^{2} \Psi}{\partial h_{g} \partial h_{1}}\right|_{(e, 0,1)} & \cdots & \left.\frac{\partial^{2} \Psi}{\partial h_{g}^{2}}\right|_{(e, 0,1)}
\end{array}\right]\right) \\
& =\operatorname{det}\left(\left[\begin{array}{ccc}
\left.\frac{\partial^{2} \tilde{\psi}}{\partial h_{1}^{2}}\right|_{e} & \cdots & \left.\frac{\partial^{2} \tilde{\psi}}{\partial h_{1} \partial h_{g}}\right|_{e} \\
\vdots & \ddots & \vdots \\
\left.\frac{\partial^{2} \tilde{\psi}}{\partial h_{g} \partial h_{1}}\right|_{e} & \cdots & \left.\frac{\partial^{2} \tilde{\psi}}{\partial h_{g}^{2}}\right|_{e}
\end{array}\right]\right),
\end{aligned}
$$

where $\tilde{\psi}: U \rightarrow \mathbb{C}$ is the function $g \mapsto \psi\left(\tilde{\mu}_{g^{-1}}(x), x\right)$. That $(e, 0,1)$ is a nondegenerate critical point then follows from the following

Lemma 2. The Hessian of $\tilde{\psi}$ at e, $H(\tilde{\psi})_{e}$, is nonsingular.

Proof. We first produce an appropriate set of local holomorphic coordinates on $M$ in the neighbourhood on $p=\pi(x)$ and on $L^{*}$ in the neighbourhood of $x$, in terms of which the action of $G$ will be a translation. Let $M_{0}=: \Phi^{-1}(0) / G$ be the symplectic reduction of $M$. Then $M_{0}$ is an $(n-g)$ dimensional complex manifold and has an induced Kähler structure. Let $\alpha: \Phi^{-1}(0) \rightarrow M_{0}$ be the projection (a principal $G$-bundle) and set $\bar{p}=\alpha(p)$. We claim:

Lemma 3. There exist an open neighbourhood $T$ of $\bar{p}$ in $M_{0}$ and a section $\sigma: T \rightarrow \Phi^{-1}(0)$ of $\alpha$, such that $\sigma(\bar{p})=p$ and $\sigma$ is holomorphic as a map $T \rightarrow M$.

Proof. For $q \in M$, let $F_{q} \subseteq T_{q} M \otimes \mathbb{C}$ be the $+i$-eigenspace of the complex structure $J_{q} \in \operatorname{End}\left(T_{q} M\right)$.

Next, if $p \in \Phi^{-1}(0)$, recall that $T_{p}\left(\Phi^{-1}(0)\right) \subseteq T_{p} M$ is a coisotropic subspace, with symplectic complement $T_{p}\left(\Phi^{-1}(0)\right)^{\perp}=T_{p}(G \cdot p)$ [GS1].

By assumption, $\Phi$ is submersive at $p \in \Phi^{-1}(0)$. Let $B$ be an open neighbourhood of $p$ on which $\Phi$ is a submersion and the action of $G$ is locally free. Then $C=: \Phi(B)$ is an open neighbourhood of $0 \in \mathfrak{g}^{*}$. For $c \in C$, let 
$W_{c}=\Phi^{-1}(c) \cap B$. Then $W_{c}$ is a $(2 n-g)$-dimensional real submanifold of $B$. For $q \in B$ let us set $R_{q}=: T_{q}\left(W_{\Phi(q)}\right)^{\perp}$, the symplectic complement of $T_{q}\left(W_{\Phi(q)}\right)$.

By Lemma 3.6 of $\left[\mathbf{G S 1}\right.$ ], we have $F_{p} \cap\left(R_{p} \otimes \mathbb{C}\right)=0$; therefore, perhaps after restricting $B$ to a smaller open neighbourhood of $p$, we have $F_{q} \cap$ $\left(R_{q} \otimes \mathbb{C}\right)=0$ for all $q \in B$.

It follows that $F_{q}^{\prime}=: F_{q} \cap\left(T_{q}\left(W_{\Phi(q)}\right) \otimes \mathbb{C}\right)$ is a complex distribution on $B$, of complex rank $n-g$. Being the intersection of two integrable distributions, it is itself integrable.

Thus we may apply the complex Frobenius integrability theorem: there are local holomorphic coordinates $r_{i}$ on $B \subseteq M$ centered at $p$ such that $F^{\prime}=\left\{\partial / \partial r_{j}: j=1, \ldots, n-g\right\}$. Lemma 3 follows from this.

Let us now return to the proof of Lemma 2 . Let $z_{1}, \ldots, z_{n-g}$ be local holomorphic coordinates on $M_{0}$ centered at $\bar{p}$, and defined on an open neighbourhood $T \subseteq M_{0}$ of $\bar{p}$. We may assume that the $z_{i}$ 's are induced by a biholomorphic diffeomorphism $\chi: B_{n-g}(0,1) \rightarrow T$, where $B_{m}(w, r)$ denotes the ball centered at the origin and of radius $r>0$ in $\mathbb{C}^{m}$.

Let $\mathfrak{g}_{c}=: \mathfrak{g} \otimes \mathbb{C}$ be the Lie algebra of the complexification $\tilde{G}$ of $G$. Let $S \subseteq \mathfrak{g}_{c}$ be an open neighbourhood of 0 on which the $\operatorname{exponential~map~} \exp _{\tilde{G}}$ : $\mathfrak{g}_{c} \rightarrow \tilde{G}$ restricts to a biholomorphic diffeomorphism $S \rightarrow S^{\prime}=: \exp _{\tilde{G}}(S)$. Upon choosing an appropriate basis of $\mathfrak{g}$, which identifies $\mathfrak{g}_{c}$ with $\mathbb{C}^{g}$, we may assume that $S$ gets identified with $B_{g}(0,1)$. We now define a holomorphic chart on $M$ on an open neighbourhood $D$ of $p$ by setting

$$
\gamma: B_{n-g}(0,1) \times B_{g}(0,1) \subseteq \mathbb{C}^{n} \longrightarrow M, \quad(w, z) \mapsto \exp _{\tilde{G}}(w) \cdot \sigma(\chi(z)) .
$$

Let us write $w=a+i b$ and $z=c+i d$, for $w \in \mathbb{C}^{g}$ and $z \in \mathbb{C}^{n-g}$, with $a, b \in \mathbb{R}^{g}$ and $c, d \in \mathbb{R}^{n-g}$, and view $a, b, c, d$ as real local coordinates on $M$ in the neighbourhood of $p$. If $\xi \in \mathfrak{g}$ has coordinates $a=\left(a_{1}, \ldots, a_{g}\right)^{t} \in \mathbb{R}^{g}$ in the chosen basis of $\mathfrak{g}$, we have

$$
\gamma^{-1}\left(\exp _{G}(t \xi) \cdot p\right)=(t a, 0)
$$

We now construct local holomorphic coordinates on $L^{*}$ centered at $x$. By $G$-invariance, $L, h_{L}, \nabla_{L}$ descend to objects $L_{0}, h_{0}, \nabla_{0}$ on $M_{0}$. More precisely, with obvious notation, if $\iota: \Phi^{-1}(0) \hookrightarrow M_{0}$ is the inclusion, we have $\iota^{*}\left(L, h_{L}, \nabla_{L}\right)=\alpha^{*}\left(L_{0}, h_{0}, \nabla_{0}\right)$. We choose a local holomorphic frame $e_{L_{0}}$ for $L_{0}$ at $\bar{p}$, which perhaps after restriction we may assume defined on the open neighbourhood $T$ of Lemma 3. We shall view this as a holomorphic section of $L$ defined on the complex submanifold $\alpha(T)$ of $M$, and may assume with this identification that $e_{L_{0}}(\bar{p})=x$. Using the holomorphic action of $\tilde{G}$ on $L$, we may then extend $e_{L_{0}}$ to a section $e_{L}$ of $L$ over the open neighbourhood $D$ of $p$ in $M$ by setting

$$
e_{L}\left(\exp _{\tilde{G}}(w) \cdot \sigma(\bar{q})\right)=\exp _{\tilde{G}}(w) \cdot e_{L_{0}}(\sigma(\bar{q})) \quad(w \in S, \bar{q} \in T) .
$$


Let $e_{L}^{*}$ be the dual frame. The choice of $e_{L}^{*}$ then induces a holomorphic chart

$$
\tilde{\gamma}: B_{n-g}(0,1) \times B_{g}(0,1) \times \mathbb{C} \longrightarrow L_{D}^{*}=:\left.L^{*}\right|_{D} .
$$

We have, with $\xi \in \mathfrak{g}$ and $a \in \mathbb{R}^{g}$ as above,

$$
\tilde{\gamma}^{-1}\left(\exp _{\tilde{G}}(t \xi) \cdot x\right)=(t a, 0,1) .
$$

Let now $\beta=:\left\|e_{L}^{*}\right\|^{2}$, so that $\beta^{-1 / 2} e_{L}^{*}$ is a unitary frame of $L^{*}$ over $D$. We have an induced trivialization

$$
\hat{\gamma}: B_{n-g}(0,1) \times B_{g}(0,1) \times S^{1} \longrightarrow X_{D}^{*}=:\left.X^{*}\right|_{D},
$$

and since the action of $G$ preserves the metric we still have, with $\xi \in \mathfrak{g}$ and $a \in \mathbb{R}^{g}$ as above,

$$
\hat{\gamma}^{-1}\left(\exp _{\tilde{G}}(t \xi) \cdot x\right)=(t a, 0,1) .
$$

We now estimate $\psi\left(\tilde{\mu}_{\exp _{G}(-t \xi)}(x), x\right)$ to second order using the Taylor expansion of $\psi$ at $(x, x),(25)$ and (13). Since $\xi_{X}(x)=\left.\sum_{t=1}^{g} a_{t}^{\prime} \frac{\partial}{\partial a_{i}}\right|_{x}$ is horizontal in view of (19) and because $x \in \Phi^{-1}(0)$, it is annihilated by the connection form $\alpha_{x}$. Thus $d_{(x, x)} \psi((a, 0))=0$, and we have

$$
\begin{gathered}
\psi\left(\tilde{\mu}_{\exp _{G}(-t \xi)}(x), x\right)=\psi(x, x)+\frac{1}{2} t^{2} a^{t} H(\tilde{\psi})_{e} a+O\left(t^{3}\right) \\
=\psi(x, x)+\frac{1}{2} t^{2} H(\rho)_{X}^{(2,0)}\left(\xi_{X}(x)\right)+O\left(t^{3}\right), \quad(t \in \mathbb{R}) .
\end{gathered}
$$

where

$$
H(\rho)_{x}^{(2,0)}=\frac{1}{2} \sum_{i, j} \frac{\partial^{2} \rho}{\partial z_{i} \partial z_{j}}(x) d z_{i} d z_{j} .
$$

Thus Lemma 2 may be rephrased as:

Lemma 4. The quadratic form $\alpha_{x} \in \operatorname{Sym}^{2}\left(\mathfrak{g}^{*} \otimes \mathbb{C}\right)$ given by

$$
\alpha_{x}: \xi \in \mathfrak{g} \mapsto H(\rho)_{x}^{(2,0)}\left(\xi_{X}(x)\right)
$$

is nondegenerate (clearly $\xi_{L^{*}}(x)=\xi_{X}(x)$ when $\left.x \in X\right)$.

Proof of Lemma 4. Let $H(\rho)_{x}$ be the Hessian of $\rho$ at $x \in X \subset L^{*}$, in local holomorphic coordinates induced by some holomorphic trivialization of $L$ in the neighbourhood of $p=\pi(x)$; we may decompose it into types, as

$$
H(\rho)_{x}=H(\rho)_{x}^{(2,0)}+H(\rho)_{x}^{(1,1)}+H(\rho)_{x}^{(0,2)},
$$

where

$$
H(\rho)_{x}^{(1,1)}=\sum_{i, j} \frac{\partial^{2} \rho}{\partial z_{i} \partial \bar{z}_{j}}(x) d z_{i} d \bar{z}_{j}, \quad H(\rho)_{x}^{(0,2)}=\frac{1}{2} \sum_{i, j} \frac{\partial^{2} \rho}{\partial \bar{z}_{i} \partial \bar{z}_{j}}(x) d \bar{z}_{i} d \bar{z}_{j} .
$$


Since $\rho$ is $G$-invariant we have, for any $\xi \in \mathfrak{g}$ :

$$
\begin{aligned}
0 & =H(\rho)_{x}\left(\xi_{X}(x)\right) \\
& =H(\rho)_{x}^{(2,0)}\left(\xi_{X}(x)\right)+H(\rho)_{x}^{(1,1)}\left(\xi_{X}(x)\right)+H(\rho)_{x}^{(0,2)}\left(\xi_{X}(x)\right) \\
& =H(\rho)_{x}^{(1,1)}\left(\xi_{X}(x)\right)+2 \operatorname{Re}\left(H(\rho)_{x}^{(2,0)}\left(\xi_{X}(x)\right)\right),
\end{aligned}
$$

as $\rho$ is real-valued. By (19), if $x \in \Phi^{-1}(0)$ then $\xi_{X}(x)$ is horizontal, that is, it lies in the maximal complex subspace $H_{x}(X / M)$ of $T_{x}(X) \subset T_{x}\left(L^{*}\right)$. By the ampleness of $L$, the Levi form $L_{x}(\rho)$ induces a positive definite Hermitian form on $H_{x}(X / M)$. In turn, because $G$ acts freely on $\Phi^{-1}(0)$, the induced real quadratic form on $\mathfrak{g}$,

$$
\xi \mapsto L_{x}(\rho)\left(\xi_{X}(x)\right)=: H(\rho)_{x}^{(1,1)}\left(\xi_{X}(x)\right),
$$

is positive definite. This proves Lemma 4 , because a complex symmetric matrix with negative definite real part is nondegenerate.

Hence $(e, 0,1)$ is a nondegenerate critical point of $\Psi$. We still have to prove that, perhaps after restricting $U$ to a smaller open neighbourhood of $e \in G$, it is the only critical point of $\Psi$ on $U \times S^{1} \times(0, \infty)$.

Now $(0,1)$ is the only critical point of $\Psi(x, e, \theta, u)$ as a function of $(\theta, u)$. Therefore, given any $\epsilon>0$ we may replace $U$ with some possibly smaller open neighbourhood of $e$, so that $(e, 0,1)$ is the only critical point of $\Psi$ in $U \times S^{1} \times(1-\epsilon, 1+\epsilon)$. Suppose that there is a sequence $\left(g_{i}, \theta_{i}, t_{i}\right)$ of critical points of $\Psi$ with $g_{i} \rightarrow e$. Since $S^{1}$ is compact, the sequence $\theta_{i}$ has some accumulation point $\theta_{\infty}$, and after passing to a subsequence this implies $(d \Psi)_{\left(e, \theta_{\infty}, t_{i}\right)} \rightarrow 0$. By $(22)$, we must have $\theta_{\infty}=0$ and $t_{i} \rightarrow 1$, against the fact that $\left|t_{i}-1\right| \geq \epsilon$.

This completes the proof of the Lemma.

We are now in a position to apply the complex stationary phase Lemma to estimate the asymptotic behaviour of $(17)$ as $k \rightarrow+\infty[\mathbf{H}],[\mathbf{M S}]$. More precisely, since $\chi_{\omega}(e)=\operatorname{dim}\left(V_{\omega}\right)$,

$$
\begin{array}{r}
\Pi_{k, \omega}(x, x)^{\prime} \sim k^{n-g / 2} \operatorname{dim}\left(V_{\omega}\right)^{2} \operatorname{det}(H(\Psi)(e, 0,1) / 2 \pi i)^{-1 / 2} s_{0}(x, x) \\
+O\left(k^{n-g / 2-1}\right) .
\end{array}
$$

Theorem 1 follows in view of (11), (24) and Lemma 2.

\section{Proof of Theorem 2.}

We have

$$
\operatorname{dim} H^{0}\left(M, L^{\otimes k}\right)_{\omega}=\int_{X} \Pi_{\omega, k}(x, x) d x,
$$

and we want to estimate asymptotically the latter integral as $k \rightarrow+\infty$. 
As we have seen, the equivariant Szegö kernel $\Pi_{\omega, k}$ is given by

$$
\Pi_{\omega, k}(x, y)=\frac{1}{2 \pi} \int_{0}^{2 \pi} \int_{G} e^{-i k \theta} \chi_{\omega}\left(g^{-1}\right) \Pi\left(e^{i \theta} \mu_{g^{-1}}(x), y\right) d \theta d g \quad(x, y \in X) .
$$

Here $\mu$ is the action of $G$ on $X$, and $\rho$ is induced by $\mu$ by the formula $\rho_{g}(f)(x)=f\left(\mu_{g^{-1}}(x)\right)(x \in X, g \in G)$.

By compactness and the results of $[\mathbf{B S}]$, we may find $\varepsilon_{0}>0$ with the following property: For any $p \in M$ and $\varepsilon>0$, let $B(p, \varepsilon)$ be the open ball in $M$ centered at $p$ and radius $\varepsilon$, in the geodesic distance on $M$. Then on the inverse image $(\pi \times \pi)^{-1}\left(B\left(p, 2 \varepsilon_{0}\right) \times B\left(p, 2 \varepsilon_{0}\right)\right)$ the Szegö kernel is microlocally equivalent to a Fourier integral operator of the type (9).

We shall denote by $\operatorname{dist}_{M}$ the geodesic distance function on $M$, as well as its pull-back to $X$, and by dist ${ }_{X}$ the geodesic distance function on $X$. Here the Riemannian metric on $X$ is defined in the natural manner in terms of the metric on $M$ and the connection, so that the projection $\pi: X \rightarrow M$ is a Riemannian submersion; $\operatorname{dist}_{X}$ is clearly $S^{1}$-invariant. Let us set:

$$
\mathcal{V}=\left\{(x, g) \in X \times G: \operatorname{dist}_{M}\left(x, \mu_{g^{-1}}(x)\right)<\epsilon_{0}^{2}\right\} .
$$

$\mathcal{V}$ is an $S^{1}$-invariant open neighbourhood of $X \times\{e\}$ in $X \times G$. We now decompose the integral (28) as follows:

$$
\begin{aligned}
\int_{X} & \Pi_{\omega, k}(x, x) d x= \\
& =\frac{1}{2 \pi} \int_{X} \int_{G} \int_{0}^{2 \pi} e^{-i k \theta} \chi_{\omega}\left(g^{-1}\right) \Pi\left(e^{i \theta} \mu_{g^{-1}}(x), x\right) d \theta d g d x \\
& =\iint_{\mathcal{V}} \chi_{\omega}\left(g^{-1}\right) \Pi_{k}\left(\mu_{g^{-1}}(x), x\right) d x d g \\
& +\iint_{X \times G \backslash \mathcal{V}} \chi_{\omega}\left(g^{-1}\right) \Pi_{k}\left(\mu_{g^{-1}}(x), x\right) d x d g \\
& =H_{1}^{(\omega, k)}+H_{2}^{(\omega, k)} .
\end{aligned}
$$

$H_{1}^{(\omega, k)}$ and $H_{2}^{(\omega, k)}$ are defined by the latter equality. $H_{2}^{(\omega, k)}$ is $O\left(k^{-N}\right)$ for every $N=1,2, \ldots$, since the Szegö kernel is smoothing away from the diagonal. We thus need to estimate $H_{1}^{(\omega, k)}$ asymptotically as $k \rightarrow+\infty$.

To this end, we shall first of all construct an appropriate finite open cover of $M$. Choose points $p_{j} \in \Phi^{-1}(0)\left(1 \leq j \leq r_{0}\right.$ for some fixed $\left.r_{0} \geq 1\right)$ such that

$$
\Phi^{-1}(0)_{\varepsilon_{0} / 2} \subseteq \bigcup_{j=1}^{r_{0}} B\left(p_{j}, \varepsilon_{0}\right) ;
$$

here $\Phi^{-1}(0)_{\varepsilon_{0} / 2}$ is the $\varepsilon_{0} / 2$-neighbourhood of $\Phi^{-1}(0)$ in $M$ in the geodesic distance. 
Let us further choose points $p_{j} \in M \backslash \Phi^{-1}(0)_{\varepsilon_{0} / 2}\left(r_{0}+1 \leq j \leq r_{1}\right.$ for some integer $r_{1}>r_{0}$ ), such that

$$
M \backslash \Phi^{-1}(0)_{\varepsilon_{0} / 2} \subseteq \bigcup_{j=r_{0}+1}^{r_{1}} B\left(p_{j}, \varepsilon_{0} / 3\right) .
$$

Clearly, $r_{0}$ and $r_{1}$ depend on $\epsilon_{0}$.

Let us then set $V_{j}=B\left(p_{j}, \varepsilon_{0}\right)$ if $1 \leq j \leq r_{0}, V_{j}=B\left(p_{j}, \varepsilon_{0} / 3\right)$ if $r_{0}+1 \leq$ $j \leq r_{1}$. This is a finite open cover of $M$. Let $1=\sum_{j} \phi_{j}$ be a partition of unity subordinate to the cover $\left\{V_{j}\right\}$. We shall also write $\phi_{j}$ for $\phi_{j} \circ \pi$. Thus, $1=\sum_{j} \phi_{j}$ will be implicitly seen as a partition of unity on $X$ subordinate to the open cover $\tilde{V}_{j}$, where $\tilde{V}_{j}=\pi^{-1}\left(V_{j}\right)$.

$H_{1}^{(\omega, k)}$ may be decomposed as

$$
\begin{array}{r}
H_{1}^{(\omega, k)}=\sum_{j} \iint_{\mathcal{V}_{j}} \chi_{\omega}\left(g^{-1}\right) \phi_{j}(x) \Pi_{k}\left(\mu_{g^{-1}}(x), x\right) d x d g \\
=\sum_{j} A_{j}^{(\omega, k)}
\end{array}
$$

where $\mathcal{V}_{j}=\left\{(x, g) \in \mathcal{V}: x \in \tilde{V}_{j}\right\}$ and $A_{j}^{(\omega, k)}$ is defined by the latter equality. We shall next estimate each $A_{j}^{(\omega, k)}$ separately.

By construction, if $(x, g) \in \mathcal{V}$ then the neighbourhood of $\left(\mu_{g^{-1}}(x), x\right)$ we may represent $\Pi$ as a Fourier integral of the type (9).

More precisely, by our choice of $\varepsilon_{0}$, for every $j=1, \ldots, r_{1}$ we may choose a preferred holomorphic section of $L$ at $p_{j}$ and adapted local holomorphic coordinates at $p_{j}$, in the sense of $[\mathbf{S Z}]$, both defined on $B\left(p_{j}, \varepsilon_{0}\right)$. We then have induced holomorphic coordinates on $L$. Let $a_{j}=a_{j}(z)$ be the square norm in this preferred frame, and let $a_{j}(z, w)$ be its extension to $M \times M$, almost analytic in $z$, and almost antianalytic in $w$. This determines a phase function $\hat{\psi}_{j}(z, \lambda, w, \mu)=i\left(1-a_{j}(z, w) \lambda \bar{\mu}\right)$, restricting to $\psi_{j}=i\left(1-\frac{a_{j}(z, w)}{\sqrt{a_{j}(z)} \sqrt{a_{j}(w)}} \lambda \bar{\mu}\right)$ on $X \times X$ (with $\left.\lambda, \mu \in S^{1}\right) . \quad \psi_{j}$ locally parametrizes the almost analytic Lagrangian relation associated to the Szegö kernel. In particular,

$$
d_{(x, x)} \psi=\left(\partial_{z} \psi(x, x), \bar{\partial}_{w} \psi(x, x)\right)=\left(\alpha_{x},-\alpha_{x}\right),
$$

for every $x \in \tilde{V}_{j}$. A straighforward computation then gives

$$
d_{\left(e^{i \theta} x, x\right)} \psi=\left(e^{i \theta} \alpha_{e^{i \theta} x},-e^{i \theta} \alpha_{x}\right) \quad\left(x \in \tilde{V}_{j}, e^{i \theta} \in S^{1}\right) .
$$


Let $s$ be the classical symbol appearing in the local representation of $\Pi$ as a Fourier integral operator. Setting $s_{j}(y, x, t)=\phi_{j}(x) s_{j}(y, x, t)$, we obtain

$$
\begin{aligned}
& A_{j}^{(\omega, k)}=\frac{1}{2 \pi} \iint_{\mathcal{V}_{j}} \int_{0}^{2 \pi} \int_{0}^{+\infty} e^{-i k \theta} \chi_{\omega}\left(g^{-1}\right) e^{i t \psi_{j}\left(e^{i \theta} \mu_{g^{-1}}(x), x\right)} \\
& \times s_{j}\left(e^{i \theta} \mu_{g^{-1}}(x), x, t\right) d x d \theta d g d t .
\end{aligned}
$$

With the change of variables $t=k u$, this is

$$
\begin{aligned}
& A_{j}^{(\omega, k)}=\frac{k}{2 \pi} \iint_{\mathcal{V}_{j}} \int_{0}^{2 \pi} \int_{0}^{+\infty} e^{i k\left(u \psi_{j}\left(e^{i \theta} \mu_{g^{-1}}(x), x\right)-\theta\right)} \chi_{\omega}\left(g^{-1}\right) \\
& \times s_{j}\left(e^{i \theta} \mu_{g^{-1}}(x), x, k u\right) d x d \theta d g d u \\
& =\frac{k}{2 \pi} \iint_{\mathcal{V}_{j}} \int_{0}^{2 \pi} \int_{0}^{+\infty} e^{i k \Psi_{j}(x, g, \theta, u)} \chi_{\omega}\left(g^{-1}\right) \\
& \times s_{j}\left(e^{i \theta} \mu_{g^{-1}}(x), x, k u\right) d x d \theta d u d g,
\end{aligned}
$$

where $\Psi_{j}: \mathcal{V}_{j} \times S^{1} \times \mathbb{R}_{+} \rightarrow \mathbb{C}$ is defined by

$$
\Psi_{j}(x, g, \theta, u)=u \psi_{j}\left(e^{i \theta} \mu_{g^{-1}}(x), x\right)-\theta .
$$

To estimate $A_{j}^{(\omega, k)}$, we shall now use the complex stationary phase Lemma. For $x \in \tilde{V}_{j}$, let $\Psi_{x}: U_{x} \times S^{1} \times(0,+\infty) \rightarrow \mathbb{C}$ be the partial function $\Psi_{j x}(g, \theta, u)=: \Psi_{j}(x, g, \theta, u)$; here $U_{x} \subseteq G$ is a suitable neigbourhood of $e \in G$.

Lemma 5. For all sufficiently small $\varepsilon_{0}$, there exists $\gamma_{0}>0$ (independent of $\left.\epsilon_{0}\right)$ such that $\left\|d \Psi_{j x}\right\| \geq \gamma_{0} \epsilon_{0}$ at any $(x, g, \theta, u) \in \mathcal{V}_{j} \times S^{1} \times \mathbb{R}_{+}$with $r_{0}+1 \leq j \leq r_{1}$.

Proof. To begin with, we make the following remark. Given any $a, b$ with $0<a<1<b$, there exists $d>0$ with the following property: For all sufficiently small $\epsilon_{0}>0$ and $(x, g, \theta, u)$ with $(x, g) \in \mathcal{V}$, we have $\left\|d \Psi_{x}\right\|>d$ if $u \notin(a, b)$.

If not, there would exist a sequence $\left(x_{i}, g_{i}, \theta_{i}, u_{i}\right)$ with

$$
\operatorname{dist}_{M}\left(\mu_{g_{i}^{-1}}\left(x_{i}\right), x_{i}\right) \rightarrow 0
$$

$u_{i} \notin(a, b)$, and $d_{\left(x_{i}, g_{i}, \theta_{i}, u_{i}\right)} \Psi_{j x} \rightarrow 0$. Given the compactness of $X$ and $G$, we may pass to a subsequence and assume $x_{i} \rightarrow x_{\infty}, g_{i} \rightarrow g_{\infty}$. Then $\mu_{g_{\infty}^{-1}}\left(x_{\infty}\right)=e^{i \vartheta_{\infty}} x_{\infty}$, for some $\vartheta_{\infty} \in[0,2 \pi)$, and $d_{\left(x_{\infty}, e^{i \vartheta_{\infty}} x_{\infty}, \theta_{i}, u_{i}\right)} \Psi_{j x} \rightarrow 0$. But by the analysis in $[\mathbf{Z}]$ of the critical points of $\psi$ this implies $\theta_{i} \rightarrow-\vartheta_{\infty}$, $u_{i} \rightarrow 1$, absurd. Thus, we may assume $u \in[a, b]$.

We shall then fix some $a \in(0,1)$ and suppose that $\epsilon_{0}$ is sufficiently small for the conclusion of Lemma 5 to hold. 
If $(x, g) \in \mathcal{V}_{j}$, let $\vartheta=\vartheta(x, g) \in[0,2 \pi)$ be uniquely determined by the condition that

$$
\operatorname{dist}_{X}\left(e^{i \vartheta} \mu_{g^{-1}}(x), x\right)=\operatorname{dist}_{M}\left(\mu_{g^{-1}}(x), x\right)<\epsilon_{0}^{2} .
$$

Given that $d_{\left(e^{i \theta} x, x\right)} \psi_{j}=\left(e^{i \theta} \alpha_{e^{i \theta} x},-e^{i \theta} \alpha_{x}\right)$ for all $x \in \tilde{V}_{j}$, there exists $C_{1}>0$ such that

$$
(x, g) \in \mathcal{V}_{j} \Longrightarrow
$$

Suppose now that $r_{0}+1 \leq j \leq r_{1}$ and $p \in V_{j}$. Then

$$
\operatorname{dist}\left(p, \Phi^{-1}(0)\right) \geq \operatorname{dist}\left(p_{j}, \Phi^{-1}(0)\right)-d\left(p, p_{j}\right) \geq \varepsilon_{0} / 6 .
$$

Suppose $x \in \tilde{V}_{j}$ and $(x, g) \in \mathcal{V}$, and set $p=\pi(x) \in V_{j}$. Then

$$
\operatorname{dist}_{M}\left(\mu_{g^{-1}}(p), p\right)=\operatorname{dist}_{M}\left(\mu_{g^{-1}}(x), x\right)<\varepsilon_{0}^{2} \text {. }
$$

Therefore,

$$
\operatorname{dist}_{M}\left(\mu_{g^{-1}}(p), \Phi^{-1}(0)\right) \geq \varepsilon_{0} / 6-\varepsilon_{0}^{2} \geq \varepsilon_{0} / 7,
$$

if $\varepsilon_{0}<1 / 42$. Thus, given that $0 \in \mathfrak{g}^{*}$ is a regular value of the moment map, there exists $C_{2}>0$ such that

$$
r_{0}+1 \leq j \leq r_{1},(x, g) \in \mathcal{V}_{j} \Longrightarrow\left\|\Phi\left(\mu_{g^{-1}}(p)\right)\right\| \geq C_{2} \varepsilon_{0},
$$

in a given fixed norm on $\mathfrak{g}^{*}$. In other words, if $r_{0}+1 \leq j \leq r_{1}$ and $(x, g) \in \mathcal{V}_{j}$ then there exists $\xi \in \mathfrak{g}^{*}$ of unit norm such that $\Phi_{\xi}\left(\mu_{g^{-1}}(p)\right) \geq C_{2} \varepsilon_{0}$, where $p=\pi(x)$ and $\Phi_{\xi}=\langle\Phi, \xi\rangle$. This is equivalent to the condition

$$
\alpha_{\mu_{g^{-1}}(x)}\left(\xi_{X}\left(\mu_{g^{-1}}(x)\right)\right) \geq C_{2} \varepsilon_{0},
$$

and in view of the above this completes the proof of Lemma 5 .

We now apply the complex stationary phase Lemma to estimate the sum $\sum_{j=r_{0}+1}^{r_{\epsilon}} H_{3, j}^{(\omega, k)}$. Recalling the definition of $H_{3, j}^{(\omega, k)}$, we first integrate over $\theta, u, g$ and then over $x$. The first integral, given Lemma 5 , is $\leq C_{N} k^{-N}$ for every $N=1,2, \ldots$. We thus obtain

$$
\left|\sum_{j=r_{0}+1}^{r_{\epsilon}} H_{3, j}^{(\omega, k)}\right| \leq C_{N} k^{-N} \int_{X}\left(\sum_{j=r_{0}+1}^{r_{\epsilon}} \phi_{j}\right) d x \leq C_{N}^{\prime} k^{-N} .
$$

In order to estimate $\sum_{j=1}^{r_{0}} H_{3, j}^{(\omega, k)}$, we shall use in the neighbourhood of any $x \in \pi^{-1}\left(\Phi^{-1}(0)\right)$ the local coordinates $\left(w, z, \lambda=e^{i \theta}\right)$ discussed in the proof of Lemma 2. Thus $w \in \mathbb{C}^{g}, z \in \mathbb{C}^{n-g}$ and $\lambda \in S^{1}$. We shall write $w=a+i b$ and $z=c+i d$, where $a, b \in \mathbb{R}^{g}$ and $c, d \in \mathbb{R}^{n-g}$. Then $\pi^{-1}\left(\Phi^{-1}(0)\right)$ is locally defined near $x$ by the equation $\{b=0\}$. In the neighbourhood of $(x, e, 1,1) \in X \times G \times S^{1} \times \mathbb{R}$ we then have local coordinates 
$\left(a, b, c, d, \lambda=e^{i \theta}, a^{\prime}, \lambda^{\prime}=e^{i \theta^{\prime}}, u\right)$. In the following we shall not distinguish between $\Psi$ and its expression in local coordinates.

Loosely speaking, $\pi^{-1}\left(\Phi^{-1}(0)\right) \times\{(e, 1,1)\}$ is a nondegenerate critical manifold for the (locally defined) phase $\Psi$.

Lemma 6. Every $x \in \pi^{-1}\left(\Phi^{-1}(0)\right) \times\{(e, 1,1)\}$ is a critical point of $\Psi$. The Hessian of $\Psi$ at any $x \in \pi^{-1}\left(\Phi^{-1}(0)\right) \times\{(e, 1,1)\}$ has rank $2 g+2$, and is nondegenerate in the variables $\left(b, a^{\prime}, \theta^{\prime}, u\right)$.

Proof. By the previous discussion, $\pi^{-1}\left(\Phi^{-1}(0)\right) \times\{(e, 1,1)\}$ is a critical manifold for $\Psi$. Thus the Hessian of $\Psi$ at any $x \in \pi^{-1}\left(\Phi^{-1}(0)\right) \times\{(e, 1,1)\}$, $H(\psi)_{x}$, is a well-defined quadratic form on the tangent space to $X \times G \times$ $S^{1} \times \mathbb{R}$ at $x$. Suppose without loss of generality that the local coordinates $\left(a, b, c, d, \lambda=e^{i \theta}\right)$ are centered at $x$. Clearly, every second derivative of $\Psi$ involving one of the variables $a, c, d, \theta$ (which give local coordinates on $\left.\pi^{-1}\left(\Phi^{-1}(0)\right) \times\{(e, 1,1)\}\right)$ vanishes at $x$ (incidentally, given the expression in local coordinates of the phase $\psi$ discussed in $[\mathbf{Z}], \Psi$ does not depend on $\lambda)$. We want to show that $H(\psi)_{x}$ is nondegenerate on the subspace

$$
\operatorname{span}\left\{\left.\frac{\partial}{\partial b_{i}}\right|_{x},\left.\frac{\partial}{\partial a_{i}^{\prime}}\right|_{x},\left.\frac{\partial}{\partial \theta^{\prime}}\right|_{x},\left.\frac{\partial}{\partial u}\right|_{x}\right\} \text {. }
$$

Let us then consider then the $(2 g+2) \times(2 g+2)$ matrix given by Hessian of $\Psi$ at $x$ in the variables $\left(b, a^{\prime}, \theta, u\right), \hat{H}(\psi)_{x}$. To begin with, let us remark that

Claim 2.1.

$$
\left.\frac{\partial^{2} \Psi}{\partial u \partial a^{\prime}}\right|_{x}=0,\left.\frac{\partial^{2} \Psi}{\partial u \partial b}\right|_{x}=0
$$

Proof. By definition, $\frac{\partial \Psi}{\partial u}=\psi$. The first vanishing then holds because $d_{(x, x)} \psi=\left(\alpha_{x},-\alpha_{x}\right)$, where $\alpha$ is the connection 1-form, and as we have seen the action of $G$ at $x \in \pi^{-1}\left(\Phi^{-1}(0)\right)$ is horizontal to first order. The second vanishing holds because $\psi$ is constant along the diagonal.

As recalled in the proof of Theorem 1, the Hessian of $\Psi$ in the two variables $\theta^{\prime}, u$ at $(0,1)$ is known from $[\mathbf{Z}]$. Given the Claim, we obtain for the Hessian in $b, a^{\prime}, \theta^{\prime}, u$ :

$\operatorname{det} \hat{H}(\Psi)_{x}=\operatorname{det}\left(\left[\begin{array}{cc}\left.\frac{\partial^{2} \Psi}{\partial b^{2}}\right|_{x} & \left.\frac{\partial^{2} \Psi}{\partial a^{\prime} \partial b}\right|_{x} \\ \left.\frac{\partial^{2} \Psi}{\partial a^{\prime} \partial b}\right|_{x} & \left.\frac{\partial^{2} \Psi}{\partial a^{\prime 2}}\right|_{x}\end{array}\right]\right)=\operatorname{det}\left(\left[\begin{array}{cc}\left.\frac{\partial^{2} \psi}{\partial b^{2}}\right|_{x} & \left.\frac{\partial^{2} \psi}{\partial a^{\prime} \partial b}\right|_{x} \\ \left.\frac{\partial^{2} \psi}{\partial a^{\prime} \partial b}\right|_{x} & \left.\frac{\partial^{2} \psi}{\partial a^{\prime 2}}\right|_{x}\end{array}\right]\right)$.

Claim 2.2.

$$
\left.\frac{\partial^{2} \Psi}{\partial b^{2}}\right|_{x}=0
$$


Proof. If we fix $g=e, \theta^{\prime}=0, u=1, \Psi=\psi$ is constant as a function of $x$.

Thus,

$$
\operatorname{det} \hat{H}(\Psi)_{x}=\operatorname{det}\left(\left[\begin{array}{cc}
0 & \left.\frac{\partial^{2} \psi}{\partial a^{\prime} \partial b}\right|_{x} \\
\left.\frac{\partial^{2} \psi}{\partial a^{\prime} \partial b}\right|_{x} & \left.\frac{\partial^{2} \psi}{\partial a^{\prime 2}}\right|_{x}
\end{array}\right]\right)
$$

and we are reduced to proving that the $g \times g$ symmetric matrix $\left.\frac{\partial^{2} \Psi}{\partial a^{\prime} \partial b}\right|_{x}=$ $\left.\frac{\partial^{2} \psi}{\partial a^{\prime} \partial b}\right|_{x}$ is nonsingular. We shall do this using the second order expansion of $\Psi=\psi$ as a function of $a^{\prime}$ and $b$ keeping fixed $a=0, \theta=\theta^{\prime}=0, u=1$. In so doing, we shall use the Taylor expansion of $\psi$ (as a function on $L^{*}$ ) at $(x, x)$; this is determined as we have seen by the Taylor expansion of the square norm function $\varrho$.

In the notation of the proof of Lemma 2, let $\gamma(a+i b, c+i d), \tilde{\gamma}(a+i b, c+$ $i d, z)$ and $\hat{\gamma}(a+i b, c+i d, \lambda)$ be, respectively, the local charts of $M, L^{*}$ and $X \subseteq L^{*}$ near $p=\pi(x)$ and $x$; here $a, b \in \mathbb{R}^{g}, c, d \in \mathbb{R}^{n-g}, z \in \mathbb{C}, \lambda \in S^{1}$. Thus,

$$
\begin{gathered}
\gamma(a+i b, 0)=\exp (a+i b) \cdot p, \\
\tilde{\gamma}(a+i b, 0, z)=\exp (a+i b) \cdot\left(p, z e_{L}^{*}(p)\right), \\
\hat{\gamma}(a+i b, c+i d, \lambda)=\tilde{\gamma}(a+i b, c+i d, \lambda / \sqrt{\beta(\gamma(a+i b, c+i d))}),
\end{gathered}
$$

where $\beta=\left\|e_{L}^{*}\right\|^{2}$. To simplify notation, we identify $a+i b \in \mathbb{C}^{g}$ with the corresponding vector in $\mathfrak{g}_{c}$, given the implicit choice of a fixed basis of $\mathfrak{g}$. We also write $g=\exp (a+i b)$ for its image in $\tilde{G}$ under the exponential map. With some further abuse of notation, in the following lines we shall omit the variables $c, d$ : for example, $\hat{\gamma}(i t b, \lambda)$ will then really mean $\hat{\gamma}(i t b, 0, \lambda)$, where $\lambda=e^{i \theta}$.

We shall also let $p=\pi(x)$, so that $\gamma(a+i b)=\exp (a+i b) \cdot p$, and $x=\left(p, \tilde{e}_{L}^{*}(p)\right)$. By construction of $\tilde{e}_{L}$, if $a^{\prime} \in \mathbb{R}^{g}$ and $t \in \mathbb{R}$ is sufficiently small, we have

$$
\exp \left(t a^{\prime}\right) \cdot\left(q, \lambda \tilde{e}_{L}^{*}(q)\right)=\left(\exp \left(t a^{\prime}\right) \cdot q, \lambda \tilde{e}_{L}^{*}\left(\exp \left(t a^{\prime}\right) \cdot q\right)\right),
$$

for every $q \in M$ near $p$ and $\lambda \in \mathbb{C}$. In particular, if $q=\gamma(i t b)=\exp (i t b) \cdot p$, we have

$$
\begin{array}{r}
e^{t a^{\prime}} \cdot \tilde{\gamma}(i t b, \lambda)=e^{t a^{\prime}} \cdot\left(\gamma(i t b), \lambda \tilde{e}_{L}^{*}(\gamma(i t b))\right) \\
=e^{t a^{\prime}} \cdot\left(e^{i t b} \cdot p, \lambda e^{i t b} \cdot \tilde{e}_{L}^{*}\left(e^{i t b} \cdot p\right)\right) \\
=e^{t a^{\prime}} \cdot e^{i t b} \cdot\left(p, \lambda \tilde{e}_{L}^{*}(p)\right) \\
=\left(e^{t a^{\prime}} e^{i t b} \cdot p, \lambda \tilde{e}_{L}^{*}\left(e^{t a^{\prime}} e^{i t b} \cdot p\right)\right) .
\end{array}
$$


Since $\exp \left(t a^{\prime}\right) \exp (i t b)=\exp \left(t\left(a^{\prime}+i b\right)+(i / 2) t^{2}\left[a^{\prime}, b\right]+O\left(t^{3}\right)\right)$, we may rewrite this as

$$
\begin{aligned}
e^{t a^{\prime}} & \cdot \tilde{\gamma}(i t b, \lambda)= \\
& =\left(e^{t\left(a^{\prime}+i b\right)+(i / 2) t^{2}\left[a^{\prime}, b\right]+O\left(t^{3}\right)} \cdot p, \lambda \tilde{e}_{L}^{*}\left(e^{t\left(a^{\prime}+i b\right)+(i / 2) t^{2}\left[a^{\prime}, b\right]+O\left(t^{3}\right)} \cdot p\right)\right) \\
& =\tilde{\gamma}\left(t\left(a^{\prime}+i b\right)+(i / 2) t^{2}\left[a^{\prime}, b\right]+O\left(t^{3}\right), \lambda\right) .
\end{aligned}
$$

We have for $a^{\prime}, b \in \mathbb{R}^{g}$ and $t \in \mathbb{R}$ sufficiently small:

$$
\begin{array}{r}
\Psi\left(\hat{\gamma}(i t b, 1), e^{-t a^{\prime}}, 0,1,\right)=\Psi\left(\tilde{\gamma}\left(i t b, \frac{1}{\beta(\gamma(i t b))}\right), e^{-t a^{\prime}}, 0,1,\right) \\
=\psi\left(e^{t a^{\prime}} \cdot\left(e^{i t b} \cdot p, \frac{1}{\beta(\gamma(i t b))}\right),\left(e^{i t b} \cdot p, \frac{1}{\beta(\gamma(i t b))}\right)\right),
\end{array}
$$

Identifying $\psi$ with its expression in local coordinates this is

$\psi\left(\left(t\left(a^{\prime}+i b\right)+(i / 2) t^{2}\left[a^{\prime}, b\right]+O\left(t^{3}\right), \frac{1}{\beta(\gamma(i t b))}\right),\left(i t b, \frac{1}{\beta(\gamma(i t b))}\right)\right)$.

Now, as we have mentioned, the action of $G$ on $X \subseteq L^{*}$ is horizontal over $\Phi^{-1}(x)$. Thus, given that $\pi(x) \in \Phi^{-1}(x)$, for every $\xi \in \mathfrak{g}$ the associated tangent vector $\xi_{L^{*}}(x)=\xi_{X}(x) \in H(X / M)_{x}$. The latter is the maximal complex subspace of $T_{x} L^{*}$ contained in $T_{x} X$. Therefore, we also have $J_{x}\left(\xi_{X}(x)\right)=(i \xi)_{X}(x) \in H(X / M)_{x}$. Now, in more intrinsic notation, suppose $a^{\prime}, b \in \mathbb{R}^{g}$ correspond to $\xi^{\prime}, \xi \in \mathfrak{g}$, respectively; then, in the local coordinates provided by the holomorphic chart $\tilde{\gamma},\left(a^{\prime}, 0\right)$ stands for $\xi_{L^{*}}^{\prime}(x)$, $(b, 0)$ for $\xi_{L^{*}}(x)$, and $(i b, 0)$ for $J_{x} \xi_{L^{*}}(x)\left(J_{x}\right.$ is the complex structure on $\left.T_{x} L^{*}\right)$. Thus, on the one hand in our local coordinates

$$
\alpha_{x}\left(t\left(a^{\prime}+i b\right)+(i / 2) t^{2}\left[a^{\prime}, b\right], 0\right)=0, \alpha_{x}(i t b, 0)=0 .
$$

On the other hand, the path $\tilde{\gamma}(i t b)$ is tangent to $X$ at $t=0$, and therefore $\beta(\gamma(i t b))^{-1}=1+t^{2} s(t)$ for some smooth function $s(t)$. Given that $d_{(x, x)} \psi=$ $\left(\alpha_{x},-\alpha_{x}\right)$, where $\alpha$ is the connection 1-form, we conclude from the latter expressions that

$$
\Psi\left(\hat{\gamma}(i t b, 1), e^{-t a^{\prime}}, 0,1,\right)=\frac{1}{2} t^{2} H(\psi)_{(x, x)}\left(a^{\prime}+i b, i b\right)+O\left(t^{3}\right) .
$$

In view of (13),

$$
\begin{gathered}
H(\psi)_{(x, x)}\left(a^{\prime}+i b, i b\right)=H^{(2,0)}(\varrho)_{x}\left(a^{\prime}+i b, a^{\prime}+i b\right) \\
+H^{(1,1)}(\varrho)_{x}\left(a^{\prime}+i b, i b\right)+H^{(0,2)}(\varrho)_{x}(i b, i b) .
\end{gathered}
$$

Since $\psi(x, x)$ vanishes identically for $x \in X$,

$$
\psi\left(\left(\gamma(i t b), \frac{1}{\beta(\gamma(i t b))}\right),\left(\gamma(i t b), \frac{1}{\beta(\gamma(i t b))}\right)\right)=0
$$


for every $t$, and therefore in (39) the terms containing only $b$ add up to zero. Using the symmetry and the $\mathbb{C}$-linearity of $H^{(2,0)}(\varrho)$ and the sesquilinearity of $H^{(1,1)}(\varrho)$, we obtain

$$
\begin{aligned}
& H(\psi)_{(x, x)}\left(a^{\prime}+i b, i b\right)= \\
& \quad=H^{(2,0)}(\varrho)_{x}\left(a^{\prime}, a^{\prime}\right)+i\left(2 H^{(2,0)}(\varrho)_{x}-H^{(1,1)}(\varrho)_{x}\right)\left(a^{\prime}, b\right) .
\end{aligned}
$$

Thus, the $g \times g$ complex matrix $-\left.i \frac{\partial^{2} \Psi}{\partial a^{\prime} \partial b}\right|_{x}$ represents the bilinear pairing $\mathfrak{g} \times \mathfrak{g} \rightarrow \mathbb{C}$ given by

$$
(\xi, \eta) \mapsto 2 H^{(2,0)}(\varrho)_{x}\left(\xi_{X}(x), \eta_{X}(x)\right)-H^{(1,1)}(\varrho)_{x}\left(\xi_{X}(x), \eta_{X}(x)\right) .
$$

The latter is in turn the restriction of a similarly defined $\mathbb{R}$-bilinear pairing on $\mathfrak{g}_{c}$. We have seen in the proof of Theorem 1 that the former term is represented by a complex symmetric matrix with negative definite real part, while the second term is a negative definite Hermitian product on $\mathfrak{g}_{c}$. The proof of Lemma 6 is then completed by the following:

Lemma 7. Let $b \geq 1$ be an integer. Let $C$ and $H$ be complex $b \times b$ matrices. Suppose that $C$ is symmetric and $H$ is Hermitian. Suppose furthermore that $C$ has positive definite real part, and that $H$ is positive semidefinite. Then $C+H$ is nonsingular.

Proof. By the hypothesis, $C=A+i B, H=R+i S$, where $A, B, R, S$ are real $b \times b$ matrices satisfying: $A^{t}=A, B^{t}=B$, and $A$ is positive definite; $R^{t}=R, S^{t}=-S$ and

$$
\left(V^{t}+i W^{t}\right)(R+i S)(V-i W) \geq 0
$$

for all $V, W \in \mathbb{R}^{b}$, and equality holds if and only if $V=W=0$. Suppose that $X+i Y \in \operatorname{ker}(C+H)$, where $X, Y \in \mathbb{R}^{b}$. We have

$(41)\left(X^{t}-i Y^{t}\right)(R+i S)(X+i Y)=X^{t} R X+Y^{t} R Y-2 X^{t} S Y \geq 0$,

for all $X, Y \in \mathbb{R}^{b}$. On the other hand,

$$
\begin{aligned}
& ((A+R)+i(B+S))(X+i Y)= \\
& \quad=((A+R) X-(B+S) Y)+i((B+S) X+(A+R) Y) .
\end{aligned}
$$

Thus, $(A+R) X=(B+S) Y$ and $(A+R) Y=-(B+S) X$. Let us multiply the first relation on the left by $X^{t}$ and the second by $Y^{t}$, and sum: we get

$$
\left(X^{t} R X+Y^{t} R Y\right)+\left(X^{t} A X+Y^{t} A Y\right)=2 X^{t} S Y .
$$

In view of (41) and the positive definiteness of $A$, we conclude $X^{t} A X=0$, $Y^{t} A Y=0$ whence $X=Y=0$. 
To complete the proof of Theorem 2, we now only need to observe that the asymptotic expansion described in the statement holds for $\sum_{j=1}^{r_{0}} H_{3, j}^{(\omega, k)}$, in view of Lemma 6 and the complex stationary phase Lemma.

\section{Proof of Theorem 3.}

To ease the exposition, let us first prove Theorem 3 under the following additional simplifying assumption:

$\omega \in \mathfrak{h}_{+}^{*}$ lies in an elementary fundamental wedge for the Hamiltonian action of $G$ on $T^{*} X \backslash\{0\}$ ([GS2], page 357).

Recall that this means that $\omega$ is a regular value of the moment map $\Psi: T^{*} X \backslash\{0\} \rightarrow \mathfrak{g}^{*}$, and that $G_{\omega} \subseteq G$ acts freely on $\Psi^{-1}(\omega)$.

To begin with, let us then first of all clarify the relation between the symplectic cone $Y$ and the submanifolds

$$
W=: \Phi^{-1}(C(\mathcal{O})) \subseteq M, Z=: \Psi^{-1}(C(\mathcal{O})) \subseteq T^{*} X \backslash\{0\} .
$$

In view of (5), if $p \in X$ and $r>0$ then

$$
\left(p, r \alpha_{p}\right) \in Y \cap \Psi^{-1}(C(\mathcal{O})) \Longleftrightarrow \pi(p) \in \Phi^{-1}(C(\mathcal{O})) .
$$

In other words, we have

Lemma 8. Let $\tilde{\pi}: Y \rightarrow M$ be the projection, $\left(x, r \alpha_{x}\right) \mapsto \pi(x)$. Then

$$
Y \cap Z=\tilde{\pi}^{-1}(W) .
$$

Furthermore, $0 \notin \Phi(M)$ clearly implies:

Lemma 9. $W \subseteq M$ is compact.

Recall, after [GS1], that $Z$ and $W$ are fibrating coisotropic submanifolds of $T^{*}(X) \backslash\{0\}$ and $M$, respectively. More precisely, there exist symplectic $\mathrm{V}$-manifolds in the sense of Satake

$$
\left(Z^{\sharp}, \Omega_{Z^{\sharp}}\right) \text { and }\left(W^{\sharp}, \Omega_{W^{\sharp}}\right),
$$

and smooth maps $p_{Z}: Z \rightarrow Z^{\sharp}, p_{W}: W \rightarrow W^{\sharp}$ whose fibres are the leaves of the corresponding null foliations. Thus, if $\iota_{Z}: Z \rightarrow T^{*}(X) \backslash\{0\}$ and $\iota_{W}: W \rightarrow M$ are the inclusions, then $\iota_{Z}^{*}\left(\Omega_{T^{*} X}\right)=p_{Z}^{*}\left(\Omega_{Z^{\sharp}}\right)$ and $\iota_{W}^{*}\left(\Omega_{M}\right)=$ $p_{W}^{*}\left(\Omega_{W^{\sharp}}\right)$, where $\Omega_{T^{*} X}, \ldots$ denote the symplectic structures of the manifolds $T^{*} X, \ldots$ The fibre of $p_{Z}$ (respectively, of $p_{W}$ ) through a point $p \in Z$ (resp., $x \in W$ ) is the orbit of a certain normal subgroup $H_{f}$ of the stabilizer subgroup $G_{f}$ under the coadjoint action, where $f=\Psi(p)$ (resp., $f=\Phi(x)$ ). The Lie algebra $\mathfrak{h}_{f}$ of $H_{f}$ is the codimension one ideal of $\mathfrak{g}_{f}=\operatorname{Lie}\left(G_{f}\right)$ given by ([GS2], page 351):

$$
\mathfrak{h}_{f}=\left\{\xi \in \mathfrak{g}_{f}:<f, \xi>=0\right\} .
$$

Let $R$ be a symplectic manifold. Given an isotropic submanifold $S \subset$ $R \times R$, we shall denote by $S^{\prime}$ the corresponding isotropic relation, that is, 
the image of $S$ under the map $\left(r_{1}, r_{2}\right) \mapsto\left(r_{1},-r_{2}\right)$. Clearly $S^{\prime}$ is an isotropic submanifold of $R \times R^{-}$, where $R^{-}$denotes $R$ with the opposite symplectic structure. The fibre products

$$
Z \times_{p_{Z}} Z \text { and } W \times_{p_{W}} W
$$

are then Lagrangian relations in $T^{*}(X \times X)$ and $T^{*}(M \times M)$, respectively. We shall in a while be interested in the composition of the conic isotropic relation $\Sigma^{\prime}$, with $\Sigma$ given by $(12)$, with $D=:\left(Z \times_{p_{Z}} Z\right)$. The previous discussion obviously implies:

Lemma 10. $\Sigma^{\prime} \circ D=\tilde{\pi}^{-1}(W) \times_{p_{Z}} \tilde{\pi}^{-1}(W)$.

More explicitly, given that $\alpha$ is $G$-invariant we have

$$
\begin{array}{r}
\tilde{\pi}^{-1}(W) \times_{p_{Z}} \tilde{\pi}^{-1}(W)=\left\{\left(\left(x, r \alpha_{x}\right),\left(y, r \alpha_{y}\right)\right): \pi(x) \in W,\right. \\
\left.y \in H_{\Phi(\pi(x))} \cdot x, r>0\right\} .
\end{array}
$$

With the standard implicit identification between sections of $L^{\otimes k}$ and $S^{1}$-equivariant funcions on $X$, let

$$
H(X)_{(\omega)}=: \bigoplus_{k \geq 0} H^{0}\left(M, L^{\otimes k}\right)_{(\omega)}=\bigoplus_{k, \ell \geq 0} H^{0}\left(M, L^{\otimes k}\right)_{\ell \omega} \subseteq H(X) \subseteq L^{2}(X),
$$

and let $P_{(\omega)}: L^{2}(X) \rightarrow H(X)_{(\omega)}$ be the orthogonal projector. If $\left\{s_{j}^{(k, \ell \omega)}\right\}$ is an orthonormal basis of $H^{0}\left(M, L^{\otimes k}\right)_{\ell \omega}$ for $k, \ell=1,2, \ldots$, the Schwartz kernel of $P_{(\omega)}$ is

$$
\tilde{P}_{(\omega)}(x, y)=\sum_{k, \ell} s_{j}^{(k, \ell \omega)}(x) \otimes \bar{s}_{j}^{(k, \ell \omega)}(y) \quad(x, y \in X) .
$$

The k-th Fourier component of $\tilde{P}_{(\omega)}$ is

$$
\tilde{P}_{(\omega), k}(x, y)=\sum_{\ell} s_{j}^{(k, \ell \omega)}(x) \otimes \bar{s}_{j}^{(k, \ell \omega)}(y) \quad(x, y \in X),
$$

and we want to estimate asymptotically the diagonal behaviour of $\tilde{P}_{(\omega), k}$ on $W=\Phi^{-1}(C(\mathcal{O}))$.

To this end, we shall describe $P_{(\omega)}$ as a Fourier integral operator with complex phase and study the geometry of the associated (almost complex) canonical relation. In fact, $P_{(\omega)}$ is the composition of two Fourier integral operators whose associated canonical relations are related to the $G$-action and to the complex structure, respectively. Namely, let

$$
L^{2}(X)_{(\omega)}=\bigoplus_{\ell \geq 1} L^{2}(X)_{\ell \omega} \subseteq L^{2}(X)
$$

and denote by $Q_{(\omega)}: L^{2}(X) \rightarrow L^{2}(X)_{(\omega)}$ the orthogonal projector. If $\Pi$ denotes, as above, the Szegö projector, then $P_{(\omega)}=Q_{(\omega)} \circ \Pi$. 
As mentioned already, $\Pi$ is an elliptic degree zero Fourier integral operator with complex phase; its almost complex Lagrangian submanifold $\tilde{C}$ is locally parametrized by the phase function $\psi$, and can be characterized geometrically as follows ([BS], Propositions 2.13 and 2.16). Let $\tilde{X}$ denote a complexification of $X[\mathbf{M S}]$, so that the natural (complex) symplectic structure on the cotangent bundle $T^{*}(\tilde{X})$ is the complexification of the symplectic structure on $T^{*}(X)$. Let $\zeta=\left\{\zeta_{j}\right\}$ be the symbol of the boundary CauchyRiemann operator $\bar{\partial}_{b}$, a smooth function on $T^{*}(X)$, and let $\tilde{\zeta}=\left\{\tilde{\zeta}_{j}\right\}$ be its almost analytic extension to $T^{*}(\tilde{X})$.

Theorem 4. ([BS]) Let $\Upsilon \subseteq T^{*}(\tilde{X}) \backslash\{0\}$ be the almost analytic submanifold defined by $\tilde{\zeta}=0$. Then $\Upsilon$ is a conic involutive submanifold of $T^{*}(\tilde{X}) \backslash$ $\{0\}$, and $\tilde{C}$ is (up to almost analytic equivalence) the unique Lagrangian submanifold of $\left(T^{*}(\tilde{X}) \backslash\{0\}\right) \times\left(T^{*}(\tilde{X}) \backslash\{0\}\right)$ contained in $\Upsilon \times \bar{\Upsilon}$ and containing the wave front (12) of $\Pi$.

Next, $Q_{(\omega)}$ is also an elliptic degree zero Fourier integral operator, with real phase however. Its associated canonical relation is described in [GS2], Theorem 6.7, in terms of the moment map $\Psi$. Namely, with $\omega$ and $C(\mathcal{O})$ as in Definition 1, assume that $\Psi$ is transversal to $C(\mathcal{O})$. The conic Lagrangian relation

$$
D \subseteq\left(T^{*}(X) \backslash\{0\}\right) \times\left(T^{*}(X) \backslash\{0\}\right)
$$

associated to $Q_{(\omega)}$ is then the fibre product $Z \times{ }_{p} Z$. To discuss the composition of $\Pi$ and $Q_{(\omega)}$, we shall view the latter as a Fourier integral operator with complex phase, whose associated almost complex canonical relation

$$
\tilde{D} \subseteq\left(T^{*}(\tilde{X}) \times\{0\}\right) \times\left(T^{*}(\tilde{X}) \backslash\{0\}\right)
$$

is simply the almost analytic extension of $D$. In the language and notation of almost analytic machinery [MS], this can be described as follows.

The moment map $\Psi: T^{*}(X) \rightarrow \mathfrak{g}^{*}$ extends almost analytically to $\tilde{\Psi}$ : $T^{*}(\tilde{X}) \rightarrow \mathfrak{g}_{c}^{*}$, where $\mathfrak{g}_{c}=\mathfrak{g} \otimes_{\mathbb{R}} \mathbb{C}$. Let $\tilde{C}(\tilde{\mathcal{O}}) \subseteq \mathfrak{g}_{c}^{*}$ be the complexification of $C(\mathcal{O}) \subseteq \mathfrak{g}^{*}$; this can be explicitly decribed as

$$
\tilde{C}(\tilde{\mathcal{O}})=\left\{\lambda \omega^{\prime}: \lambda \in \mathbb{C}^{*}, \omega^{\prime} \in \tilde{\mathcal{O}}\right\},
$$

where $\tilde{\mathcal{O}} \subseteq \mathfrak{g}_{c}^{*}$ is the coadjoint orbit of $\omega$ in $\mathfrak{g}_{c}^{*}$ under the complexification $\tilde{G}$ of $G$. Then $\tilde{\Psi}$ is transversal to $\tilde{\mathcal{O}}$, and the almost analytic submanifold $\tilde{Z}=\tilde{\Psi}^{-1}(\tilde{\mathcal{O}}) \subseteq T^{*}(\tilde{X}) \backslash\{0\}$ is the complexification of $Z$. The fibration $p: Z \rightarrow M_{\omega}^{\sharp}$ extends almost analytically to $\tilde{p}: \tilde{Z} \rightarrow \tilde{M}_{\omega}^{\sharp}$, and the fibre product

$$
\tilde{D}=: \tilde{Z} \times_{\tilde{p}} \tilde{Z} \subseteq T^{*}(\tilde{X}) \times T^{*}(\tilde{X})
$$

is the almost analytic extension of $D=Z \times{ }_{p} Z$. It is therefore a Lagrangian relation in $T^{*}(\tilde{X}) \times T^{*}(\tilde{X})$. 
Clearly, $D$ and $\Sigma$ are the sets of real points of $\tilde{D}$ and $\tilde{C}$, respectively. Set

$$
\Delta=T^{*}(X) \times \operatorname{diag}\left(T^{*}(X)\right) \times T^{*}(X)
$$

and

$$
\tilde{\Delta}=T^{*}(\tilde{X}) \times \operatorname{diag}\left(T^{*}(\tilde{X})\right) \times T^{*}(\tilde{X}) .
$$

Lemma 11. $\tilde{D} \times \tilde{C}$ and $\tilde{\Delta}$ meet transversally in $T^{*}(\tilde{X}) \times T^{*}(\tilde{X}) \times T^{*}(\tilde{X}) \times$ $T^{*}(\tilde{X})$ at every point of $(D \times \Sigma) \cap \Delta$.

Proof. All statements in the following arguments are meant to be local along the real locus. We have

$$
\begin{array}{r}
\tilde{D}=\left\{\left(w, w^{\prime}\right) \in\left(T^{*}(\tilde{X}) \backslash\{0\}\right) \times\left(T^{*}(\tilde{X}) \backslash\{0\}\right):\right. \\
\left.\Psi(w), \Psi\left(w^{\prime}\right) \in \tilde{C}(\tilde{\mathcal{O}}), \tilde{p}(w)=\tilde{p}\left(w^{\prime}\right)\right\} .
\end{array}
$$

If $\left(w, w^{\prime}\right) \in \tilde{D}$, then the tangent space to $\tilde{D}$ at $\left(w, w^{\prime}\right)$ is

$$
T_{\left(w, w^{\prime}\right)}(\tilde{D})=\left\{\left(v, v^{\prime}\right) \in T_{\left(w, w^{\prime}\right)}(\tilde{Z} \times \tilde{Z}): d_{w} \tilde{p}(v)=d_{w^{\prime}} \tilde{p}\left(v^{\prime}\right)\right\} .
$$

With $g=\operatorname{dim}(G)$, set $k=g-\operatorname{dim}(\mathcal{O})-1=\operatorname{codim}(C(\mathcal{O}))$. If $v^{\prime} \in T_{w^{\prime}}(\tilde{Z})$, the collection of all $v \in T_{w}(\tilde{Z})$ with $d_{w} \tilde{p}(v)=d_{w^{\prime}} \tilde{p}\left(v^{\prime}\right)$ is an affine space of dimension $k$.

Suppose next $y=\left(w, w^{\prime}, w^{\prime}, w^{\prime \prime}\right) \in(D \times \Sigma) \cap \Delta$. Then

$$
\begin{aligned}
& \left(T_{\left(w, w^{\prime}\right)}(\tilde{D}) \times T_{\left(w^{\prime}, w^{\prime \prime}\right)}(\tilde{C})\right) \cap T_{y}(\tilde{\Delta})= \\
& \left\{\left(v, v^{\prime}, v^{\prime}, v^{\prime \prime}\right):\left(v, v^{\prime}\right) \in T_{\left(w, w^{\prime}\right)}(\tilde{Z} \times \tilde{Z}), d_{w} \tilde{p}(v)=d_{w^{\prime}} \tilde{p}\left(v^{\prime}\right),\right. \\
& \left.\left(v^{\prime}, v^{\prime \prime}\right) \in T_{\left(w^{\prime}, w^{\prime \prime}\right)}(\tilde{C})\right\} .
\end{aligned}
$$

The condition $\left(v, v^{\prime}\right) \in T_{\left(w, w^{\prime}\right)}(\tilde{Z} \times \tilde{Z})$ may be rewritten

$$
d_{w} \tilde{\Psi}(v) \in T_{\tilde{\Psi}(w)}(\tilde{C}(\tilde{\mathcal{O}})), \quad d_{w^{\prime}} \tilde{\Psi}\left(v^{\prime}\right) \in T_{\tilde{\Psi}\left(w^{\prime}\right)}(\tilde{C}(\tilde{\mathcal{O}})) .
$$

Claim 3.1. $\tilde{C}$ is transversal to $\tilde{C}(\tilde{\mathcal{O}})$ under the map $\tilde{\gamma}:\left(w, w^{\prime}\right) \mapsto \tilde{\Psi}(w)$ (along the real locus).

Proof. Certainly $\tilde{C} \supseteq \tilde{\Sigma} \supseteq \Sigma$, where

$$
\tilde{\Sigma}=\left\{\left(p, \lambda \tilde{\alpha}_{p}, p,-\lambda \tilde{\alpha}_{p}\right): p \in \tilde{X}, \lambda \in \mathbb{C}^{*}\right\}
$$

is the complexification of $\Sigma$; here $\tilde{\alpha}$ is the complexification of the connection 1-form $\alpha$. It suffices to show that $\tilde{\Sigma}$ is transversal to $\tilde{C}(\tilde{\mathcal{O}})$ under $\tilde{\gamma}$, whence that $\Sigma$ is transversal to $C(\mathcal{O})$ under the map $\gamma:\left(w, w^{\prime}\right) \mapsto \Psi(w)$. By homogeneity of the moment map on the cotangent bundle and equality (A.11) in the Appendix to [GS1], if $p \in X$ and $r>0$ then

$$
\gamma\left(\left(p, r \alpha_{p}, p,-r \alpha_{p}\right)\right)=r \Psi\left(\left(p, \alpha_{p}\right)\right)=\Phi(p) .
$$


The statement follows from the hypothesis that $\Phi$ be transversal to $C(\mathcal{O})$.

Returning to the proof of Lemma 11, the Claim clearly implies that

$$
\operatorname{dim}_{\mathbb{C}}\left(\left(T_{\left(w, w^{\prime}\right)}(\tilde{D}) \times T_{\left(w^{\prime}, w^{\prime \prime}\right)}(\tilde{C})\right) \cap T_{y}(\tilde{\Delta})\right)=\operatorname{dim}(\tilde{C})=2 r,
$$

where $r=2 n+1=\operatorname{dim}(X)$. We have on the other hand

$$
\operatorname{dim}_{\mathbb{C}}(\tilde{C})+\operatorname{dim}_{\mathbb{C}}(\tilde{D})-\operatorname{codim}_{\mathbb{C}}(\tilde{\Delta})=2 r+2 r-2 r=2 r,
$$

and thus the statement.

Lemma 12. The projection $\Pi=\left(\pi_{1}, \pi_{4}\right)$ onto the the first and fourth factors,

$\Pi:\left(w, w^{\prime}, w^{\prime}, w^{\prime \prime}\right) \in(D \times \Sigma) \cap \Delta \mapsto\left(w, w^{\prime \prime}\right) \in\left(T^{*}(X) \backslash\{0\}\right) \times\left(T^{*}(X) \backslash\{0\}\right)$, is injective and proper.

Proof. Injectivity follows immediately from the description of $\Sigma$.

Let next $K \subset\left(T^{*}(X) \backslash\{0\}\right) \times\left(T^{*}(X) \backslash\{0\}\right)$ be a compact subset. We want to establish that $\Pi^{-1}(K)$ is compact. Now

$$
\begin{array}{r}
(D \times \Sigma) \cap \Delta=\left\{\left(h \cdot\left(p, r \alpha_{p}\right),\left(p, r \alpha_{p}\right),\left(p, r \alpha_{p}\right),\left(p,-r \alpha_{p}\right)\right):\right. \\
\left.\pi(p) \in W, r>0, h \in H_{\Phi \circ \pi(p)}\right\} .
\end{array}
$$

Here $H_{f}$ denotes the codimension one closed Lie subgroup of the stabilizer $G_{f} \subseteq G$ of an element $f \in \mathfrak{g}^{*}$ discussed on page 349 of [GS2]. The projection $K^{\prime}=q_{2}(K) \subseteq T^{*}(X) \backslash\{0\}$ of $K$ onto the second factor is a compact subset of $T^{*}(X) \backslash\{0\}$. Clearly, $\Pi^{-1}(K)$ is a closed subset of

$$
\begin{array}{r}
\left\{\left(h \cdot\left(p, r \alpha_{p}\right),\left(p, r \alpha_{p}\right),\left(p, r \alpha_{p}\right),\left(p,-r \alpha_{p}\right)\right): \pi(p) \in W, r>0,\right. \\
\left.h \in H_{\Phi \circ \pi(p)},\left(p,-r \alpha_{p}\right) \in K^{\prime}\right\}
\end{array}
$$

It thus suffices to show that the latter set is compact. It is obvious that the union $\bigcup_{\pi(p) \in W} H_{\Phi \circ \pi(p)}$ is a compact subset of $G$. Since $W$ is compact, so is $\pi^{-1}(W) \subseteq X$. Therefore the set of all $r>0$ such that $\left(p,-r \alpha_{p}\right) \in K^{\prime}$ for some $p \in \pi^{-1}(W)$ is contained in a closed interval $[a, b] \subset \mathbb{R}_{+}$. The statement follows.

REMARK 3.1. With a view to Corollary 4, we remark that the condition that $W$ is compact is not essential in the proof of Lemma 12. In fact, the projection $K^{\prime \prime} \subseteq M$ of $K^{\prime}$ under the composition $T^{*} X \rightarrow X \stackrel{\pi}{\rightarrow} M$ is at any rate a compact subset of $W$, and the condition $\pi(p) \in W$ may be replaced by the condition $\pi(p) \in K^{\prime \prime}$ in (45).

Given this, we can compose the Fourier integral operators $Q_{(\omega)}$ and $\Pi[\mathbf{M S}]$ : 
Corollary 5. $P_{(\omega)}$ is a degree zero elliptic Fourier integral operator with complex phase, associated to the almost analytic Lagrangian submanifold

$$
\Gamma=\left(\tilde{D} \circ \tilde{C}^{\prime}\right)^{\prime} \subseteq\left(T^{*}(\tilde{X}) \backslash\{0\}\right) \times\left(T^{*}(\tilde{X}) \backslash\{0\}\right) .
$$

We now characterize $\Gamma$ geometrically. Let $\Upsilon \subseteq T^{*}(\tilde{X}) \backslash\{0\}$ be as in the statement of Theorem 4 .

Lemma 13. Up to almost analytic equivalence, $\Gamma$ is uniquely determined by the properties: i) $\Gamma \subseteq\left(T^{*}(\tilde{X}) \backslash\{0\}\right) \times\left(T^{*}(\tilde{X}) \backslash\{0\}\right)$ is an almost analytic Lagrangian submanifold;

ii) $\Gamma^{\prime} \supseteq D \circ \Sigma^{\prime}$;

iii) $\Gamma \subseteq \Upsilon \times \bar{\Upsilon}$.

Proof. It is clear that $\Gamma^{\prime} \supseteq D \circ \Sigma$. Let us show that $\Gamma \subseteq \Upsilon \times \bar{\Upsilon}$. We may equivalently show that $\Gamma^{\prime}=\tilde{D} \circ \tilde{C}^{\prime} \subseteq \Upsilon \times \bar{\Upsilon}$. We have, by definition,

$$
\begin{array}{r}
\Gamma^{\prime}=\left\{\left(w, w^{\prime}\right) \in\left(T^{*}(\tilde{X}) \backslash\{0\}\right) \times\left(T^{*}(\tilde{X}) \backslash\{0\}\right): \exists w^{\prime \prime} \in T^{*}(\tilde{X})\right. \\
\text { such that } \left.\left(w, w^{\prime \prime}\right) \in \tilde{D},\left(w^{\prime \prime}, w^{\prime}\right) \in \tilde{C}\right\} .
\end{array}
$$

Since $\tilde{C} \subseteq \Upsilon \times \bar{\Upsilon}$, we have $w^{\prime} \in \bar{\Upsilon}$ for all $\left(w, w^{\prime}\right) \in \Gamma$. To show that $w \in \Upsilon$, recall that

$$
\tilde{D}=\tilde{Z} \times_{\tilde{p}} \tilde{Z} \subseteq T^{*}(\tilde{X}) \times T^{*}(\tilde{X}) .
$$

It is thus sufficient to show that if $w, w^{\prime \prime} \in \tilde{Z}, \tilde{d}\left(w^{\prime \prime}\right)=0$ and $\tilde{p}(w)=\tilde{p}\left(w^{\prime \prime}\right)$, then $\tilde{d}(w)=0$. Being the complexification of the isotropic submanifold $Z, \tilde{Z}$ is also isotropic, and the leaf of its null foliation through $p \in \tilde{Z}$ is the orbit of the complex group $\tilde{H}_{f} \subseteq \tilde{G}$. Since the action of $G$ preserves the horizontal distribution $H(X / M)$ (equivalently, the connection 1-form $\alpha$ ), the symbol $d$ of the boudary Cauchy-Riemann operator is $G$-invariant. Complexifying, the action of $\tilde{G}$ preserves the horizontal distribution $H(\tilde{X} / \tilde{M})$, whence the components $\tilde{d}_{j}$ of $\tilde{d}$ are $\tilde{G}$-invariant. Equivalently, since $d$ is $G$-invariant the Hamiltonian vector fields $X_{d_{j}}$ are in the kernel of $d p$; their complexifications $\tilde{X}_{\tilde{d}_{j}}$ 's are therefore in the kernel of $d \tilde{p}$.

To establish the Lemma, we are thus reduced to proving that if

$$
\Lambda \subset\left(T^{*}(\tilde{X}) \backslash\{0\}\right) \times\left(T^{*}(\tilde{X}) \backslash\{0\}\right)
$$

is an almost analytic Lagrangian submanifold such that

$$
\Lambda^{\prime} \supseteq D \circ \Sigma^{\prime} \text { and } \Lambda \subseteq \Upsilon \times \bar{\Upsilon}
$$

then (up to almost analytic equivalence) $\Gamma=\Lambda$.

Clearly $\Lambda^{\prime} \supseteq\left(D \circ \Sigma^{\prime}\right)^{r}$, where $\left(D \circ \Sigma^{\prime}\right)^{r}$ denotes the complexification of $D \circ \Sigma^{\prime}$. Now $\left(\left(D \circ \Sigma^{\prime}\right)^{\prime}\right)^{\sim}$ is a $2(n+1)$-dimensional almost analytic isotropic submanifold of $\left(T^{*}(\tilde{X}) \backslash\{0\}\right) \times\left(T^{*}(\tilde{X}) \backslash\{0\}\right)$. On the other hand, $\Upsilon \times \bar{\Upsilon}$ 
is the coisotropic submanifold defined by the $2 n$ equations $\tilde{d}_{j}^{(1)}=0$ and $\tilde{\tilde{d}}_{j}^{(2)}=0, j=1, \ldots, n((1)$ and (2) stand for the component of evaluation). The leaves of the null foliation of $\Upsilon \times \bar{\Upsilon}$ are then generated by the flow of the Hamiltonian vector fields $\tilde{X}_{\tilde{d}_{j}}^{(1)}$ 's and $\tilde{X}_{\tilde{d}_{j}}^{(2)}$ 's. These span at each point of $\left(\left(D \circ \Sigma^{\prime}\right)^{\prime}\right)^{\sim}$ a $2 n$-dimensional linear space which is trasversal to the tangent space of $\left(D \circ \Sigma^{\prime}\right)^{\prime}$. Hence $\Lambda$ must be the union of the leaves passing through $\left(\left(D \circ \Sigma^{\prime}\right)^{\prime}\right)^{\sim}$.

We now produce suitable local holomorphic coordinates on $M$ in the neighbourhood of $p \in W$, and on $L$ in the neighbourhood of $x \in Z$. These coordinates are adapted to the fibrating structure of $W$ and $Z$.

Lemma 14. For any $p \in W$ and any sufficiently small open neighbourhood $U \ni x$ in $W$, the image of $U$ under $p_{W}$ is an open Kähler manifold $S \subseteq W^{\sharp}$. The map $p_{U}=\left.p\right|_{U}: U \rightarrow S$ is a submersion, and $\left.\Omega\right|_{U}=p_{W}^{*}\left(\Omega_{S}\right)$, where $\Omega_{S}$ is the Kähler structure of $S$.

Proof. This is proved by a straightforward adaptation of arguments from [GS1] and [GS2]; we sketch the proof for the reader's convenience and to introduce some notation. As already recalled, by the theory in [GS2], we have the following picture: If $p \in W$ and $f=\Phi(p) \in \mathfrak{g}^{*}$, let $H_{f} \subseteq G$ be the normal closed Lie subgroup of the stabilizer $G_{f}$ of $f$ with Lie algebra (43). Then the fibre $p_{W}^{-1}(p) \subseteq W$ is the orbit of $p$ under $H_{f}$. Therefore, $p_{W}^{-1}(p)$ is diffeomorphic to the quotient $H_{f} / H(p)$, where $H(p) \subseteq H_{f}$ is the stabilizer of $p$ in $H_{f}$. Furthermore, $H(p) \subseteq H_{f}$ is a discrete subgroup of $H_{f}$. Thus, $T_{p} W$ is an coisotropic subspace of $\left(T_{p} M, \Omega_{p}\right)$, with symplectic complement $\left(T_{p} W\right)^{\perp}=T_{p}\left(H_{f} \cdot p\right) \subseteq T_{p} W$. Let us set $R_{p}=: T_{p}\left(H_{f} \cdot p\right)$.

We conclude that $p_{W}$ is a submersion and $S$ is a symplectic manifold of real dimension $2\left(n-\operatorname{dim}\left(H_{f}\right)\right)=2(n-g+\operatorname{dim}(\mathcal{O})+1)$. Furthermore, for $p \in M$ let $F_{p} \subset T_{p} M \otimes \mathbb{C}$ be the $+i$-eigenspace of the complex structure $J_{p} \in$ End $\left(T_{p} M\right)$. By Lemma 3.6 of $[\mathbf{G S 1}$,

$$
F_{p} \cap\left(R_{p} \otimes \mathbb{C}\right)=0 .
$$

For $p \in W$, we set

$$
F_{p}^{\prime}=: F_{p} \cap\left(T_{p} W \otimes \mathbb{C}\right) ;
$$

then $\left(F_{p}^{\prime}\right)^{\perp}$ has complex dimension $n+\operatorname{dim} H_{f}$ ( $n$ is the complex dimension of $M)$. Therefore, by $H_{f}$-invariance and dimension count, $F^{\prime}$ descends to a well-defined positive Lagrangian complex distribution on $S$. Integrability follows as in loc. cit., and therefore $S$ inherits compatible complex and symplectic structures.

Lemma 15. Pick any $p \in W$ and let $U$ be any sufficiently small open neighbourhood of $p$ in $W$. Set $S=p_{W}(U)$, and endow it with the holomorphic 
structure described in Lemma 14. Then there exists a map $\sigma: S \rightarrow W$ satisfying the following properties: i) $\sigma\left(p_{W}(p)\right)=p$; ii): $\sigma$ is holomorphic as a map from $S$ to $M$; iii): $\sigma$ is a section of $p_{W}$, that is, $p_{W} \circ \sigma=\mathrm{id}_{S}$.

Proof. The only property not immediately obvious is (ii). Set as above $k=g-\operatorname{dim}(\mathcal{O})-1$; we may choose local coordinates $t_{1}, \ldots, t_{g}$ for $\mathfrak{g}^{*}$ on a neighbourhood $V$ of $f=\Phi(p)$ centered at $f$ and such that $C(\mathcal{O}) \cap V$ is defined by $t_{1}=\cdots=t_{k}=0$. Let $U \subseteq \Phi^{-1}(V)$ be a sufficiently small neighbourhood of $p$. The map

$$
\psi: U \rightarrow \mathbb{R}^{k}, p^{\prime} \in U \mapsto\left(t_{1}(\Phi(p)), \ldots, t_{k}(\Phi(p)) \in \mathbb{R}^{k}\right.
$$

is a submersion, and its image a neighbourhood $D$ of $0 \in \mathbb{R}^{k}$. For $c \in U$, let $W_{c}=\psi^{-1}(c) \subset U$. Then $W_{0}=W \cap U$, and $W_{c}$ is a submanifold of $U$ for every $c \in D$. If $q \in U$, define

$$
F_{q}^{\prime}=: F_{q} \cap\left(T_{q}\left(W_{\psi(q)}\right) \otimes \mathbb{C}\right) .
$$

Let us set $R_{q}=: T_{q}\left(W_{\psi(q)}\right)^{\perp}$. Given (47), we have

$$
F_{q} \cap\left(R_{q} \otimes \mathbb{C}\right)=0
$$

for every $q \in U$ (perhaps after further restricting $U$ ). This implies that $F^{\prime}$ is a complex distribution on $U$, of rank $n-g+\operatorname{dim}(\mathcal{O})+1$. It is integrable, essentially by definition, and therefore the complex version of the Frobenius integrability theorem applies. Thus, we may find local holomorphic coordinates $r_{1}, \ldots, r_{n}$ on $U$ centered at $p$, such that $F^{\prime}=\operatorname{span}\left\{\partial / \partial r_{j}: j=1, \ldots, k\right\}$. After further restricting if necessary, the manifold $\tilde{S}=\left\{r_{k+1}=\cdots=r_{n}=\right.$ $0\}$ is then a section of $p$, as required.

The $G$-invariance of $L$ and $\nabla$ clearly implies

Lemma 16. Let $U \subseteq Z$ and $S=p(U) \subseteq M_{\omega}^{\sharp}$ be as in Lemma 14. On $S$ there are an hermitian line bundle $\left(L_{S}, h_{L_{S}}\right)$ with (unique) compatible connection $\nabla_{L_{S}}$, such that $\left.L\right|_{U}=p_{U}^{*}\left(L_{S}\right), h_{L_{S}}=p_{U}^{*}\left(h_{L}\right)$ and $\nabla_{L}=p_{U}^{*}\left(\nabla_{L_{S}}\right)$.

We have in fact $L_{S}=\sigma^{*}(L), h_{L_{S}}=\sigma^{*}\left(h_{L}\right), \nabla_{L_{S}}=\sigma^{*}\left(\nabla_{L_{S}}\right)$.

Let us now fix $p \in W$ and let $U$ be the open neighbourhood of $p$ in $W$, $S$ the open Kähler manifold and $\pi_{U}=\left.p_{W}\right|_{U}: U \rightarrow S$ be the submersion described in Lemma 14. Let $\sigma: S \rightarrow U \subseteq M$ be the holomorphic section described in Lemma 15. Set $\bar{p}=\pi_{U}(p) \in S$, and let $\left(z_{1}, \ldots, z_{k}\right)$ be local holomorphic coordinates on $S$ centered at $\bar{p}$, where $k=n-g+\operatorname{dim} \mathcal{O}+1$. Let $H_{f}^{c} \subseteq \tilde{G}$ be the complexification of $H_{f}$, with Lie algebra $\mathfrak{h}_{f}^{c}=\mathfrak{h}_{f} \otimes \mathbb{C}$. Let $\exp : \mathfrak{h}_{f}^{c} \rightarrow H_{f}^{c}$ be the exponential map of $H_{f}^{c}$, and let $V \subset \mathfrak{h}_{f}^{c}$ be an open neighbourhood of the origin such that exp induces a diffeomorphism of $V$ onto its image. Then, perhaps retricting $S$ and $V$ to smaller open subsets, the holomorphic map $\zeta: S \times V \rightarrow M$ given by

$$
\zeta:(s, v) \mapsto \exp (v) \cdot \sigma(s)
$$


is a diffeomorphism onto its image. We obtain local holomorphic coordinates $\left(z_{1}, \ldots, z_{k}, w_{1}, \ldots, w_{h}\right)$ on $M$ centered at $x$; here $w_{j}=u_{j}+i v_{j}$, where $u_{j}$ and $v_{j}$ are linear coordinates on $\mathfrak{h}_{f}$, and $h=n-k=g-\operatorname{dim} \mathcal{O}-1$.

We may also suppose without loss of generality that the local holomorphic coordinates $(z, w)$ are preferred at $p$ in the sense of $[\mathbf{S Z}]$ : in our integrable case this simply means that in the given coordinate system the Kähler form at $p$ is the standard symplectic structure $\omega_{0}$ on $\mathbb{C}^{n}$. To see this, notice that we have a symplectically orthogonal direct sum

$$
T_{p}(M) \otimes \mathbb{C}=F_{p}^{\prime} \oplus\left[R_{p} \oplus J_{p}\left(R_{p}\right)\right],
$$

where $R_{p}=T_{p}\left(H_{f} \cdot p\right)$. Furthermore, $R_{p}$ and $J_{p}\left(R_{p}\right)$ are dually paired Lagrangian subspaces of the symplectic subspace $R_{p} \oplus J_{p}\left(R_{p}\right)$. In terms of the decomposition

$$
T_{0}(S \times V)=\mathbb{C}^{k} \oplus \mathfrak{h}_{f}^{c} \text { and } \mathfrak{h}_{f}^{c}=\mathfrak{h}_{f} \oplus i \cdot \mathfrak{h}_{f}
$$

we have

$$
\begin{array}{r}
F_{p}^{\prime}=d_{0} \zeta\left(\mathbb{C}^{k} \oplus\{0\}\right), R_{p}=d_{0} \zeta\left(\{0\} \oplus \mathfrak{h}_{f} \oplus\{0\}\right), \\
J_{p}\left(R_{p}\right)=d_{0} \zeta\left(\{0\} \oplus\{0\} \oplus i \cdot \mathfrak{h}_{f}\right) .
\end{array}
$$

Thus, in order for $(z, w)$ to be a preferred system of local holomorphic coordinates at $p$ it is sufficient to choose the $z_{i}$ 's so that they form a system of preferred coordinates on $S$ at $\bar{p}$, and to make an appropriate choice for a basis in $\mathfrak{h}_{f}$.

Let now $e_{L}$ be a preferred holomorphic local frame for $L$ at $p$ in the sense of $[\mathbf{S Z}]$. Given our choice of a preferred system of local holomorphic coordinates $(z, w)$ at $p$, the function $\beta=\left\|e_{L}^{*}\right\|^{2}=\left\|e_{L}\right\|^{-2}$ then satisifes

$$
\beta(z, w)=1+|z|^{2}+|w|^{2}+\cdots .
$$

A point in the neighbourhood of $L^{*}(p) \subseteq L^{*}$ with local holomorphic coordinates $(z, w, \lambda)$ lies on $X$ if and only if $|\lambda|^{2} \beta(z, w)=1$.

In order to obtain an asymtpotic expansion for the equivariant Szëgo kernels $P_{\omega}$ on the diagonal, we shall need a more complete description of their microlocal structure near any given point of $\operatorname{diag}(W)$. To this end, we shall now prove the existence of a regular positive phase function, in the sense of [MS], microlocally associated to the Lagrangian almost analytic manifold $\Gamma$ in the neighbourhood of any point in $(p, p) \in \operatorname{diag}(W)$. The phase function will be (locally) defined on $X \times X \times \mathbb{R}^{+}$and have the form $t \cdot \psi_{\omega}(x, y)$.

Let us then fix $p_{0} \in W$ and adopt in an open neighbourhood $T$ of $p_{0}$ in $M$ the local holomorphic coordinates $(z, w)$ introduced above. This determines local holomorphic coordinates $\left(z, w, z^{\prime}, w^{\prime}\right)$ on the open neighbourhood $T \times$ $T \subseteq M \times M$ of $\left(p_{0}, p_{0}\right) \in \operatorname{diag}(W)$. Let us choose on $T$ (perhaps after restriction) a preferred holomorphic local frame $e_{L}$ for $L$ at $p_{0}$. 
Recall that $\tilde{M}$ and $\tilde{X}$ denote the almost analytic extensions of $M$ and $X$, and that the $S^{1}$-principal bundle $\pi: X \rightarrow M$ almost analytically extends to a $\mathbb{C}^{*}$-bundle $\tilde{\pi}: \tilde{X} \rightarrow \tilde{M}$.

Proposition 2. Let $p_{0} \in W$ and $T$ be as above. Then, perhaps after restricting $T$ to a smaller open neighbourhood of $p_{0}$, there exists a smooth function $b \in \mathcal{C}^{\infty}(T \times T)$ with the following property: Let $\psi \in \mathcal{C}^{\infty}\left(\left.X\right|_{T} \times\left. X\right|_{T}\right)$ be the restriction of $\hat{\psi} \in \mathcal{C}^{\infty}\left(\left.L\right|_{T} \times\left. L\right|_{T}\right)$ given by:

$$
\hat{\psi}\left(\ell, \ell^{\prime}\right)=i \cdot(1-\bar{\lambda} \mu b(p, q)),
$$

where $\ell,\left.\ell^{\prime} \in L\right|_{T}$ correspond to $(p, \lambda),(q, \mu) \in T \times \mathbb{C}$, respectively. Then $t \cdot \psi \in \mathcal{C}^{\infty}\left(\left.X\right|_{T} \times\left. X\right|_{T} \times \mathbb{R}_{+}\right)$is a regular phase function for $\Gamma$.

This means the following $[\mathbf{M S}],[\mathbf{S Z}]$. Let $\tilde{\psi}$ be the almost analytic extension of $\psi$ to $\left.\tilde{X}\right|_{\tilde{T}} \times\left.\tilde{X}\right|_{\tilde{T}}$. Then

$$
\Gamma=\left\{\left(\tilde{x}, t d_{\tilde{x}} \tilde{\psi}, \tilde{y}, t d_{\tilde{y}} \tilde{\psi}\right): \tilde{\psi}(\tilde{x}, \tilde{y})=0, t \in \mathbb{R}_{+}\right\} .
$$

In addition, we shall prove in Proposition 3 that $\psi$ is of positive type, that is, the imaginary part of $\psi$ satisfies $\Im(\psi) \geq 0$.

Proof of Proposition 2. We shall adapt the reduction argument in the proof of Theorem 2.1 of $[\mathbf{S Z}]$ to the present equivariant context.

As a first step, we shall replace the holomorphic preferred local section $e_{L}$ of $L$ at $p$ with a different local section, $\tilde{e}_{L}$, not holomorphic but capturing the equivariant geometry of our picture. We shall construct a phase function for $\Gamma$ of the asserted form $\phi=i(1-\lambda \bar{\mu} c(p, q))$ in the local (nonholomorphic) coordinates on $L^{*}$ (and $X$ ) associated to the holomorphic coordinates $(z, w)$ on $M$ and the section $\tilde{e}_{L}^{*}$. The phase function in the original holomorphic coordinates will then be given by pull-back under the change of basis from $e_{L}^{*}$ to $\tilde{e}_{L}^{*}$. Going back to holomorphic coordinates will be convenient in the proof of Proposition 3.

To this end, in the notation of Lemmas 15 and 14, let us first of all choose a local holomorphic section $e_{L_{S}}$ of the Hermitian holomorphic line bundle $L_{S}=\sigma^{*}(L)$ on $S$ which is preferred at $p_{0}$. We shall view this as a section of $L$ defined on the submanifold $\sigma(S) \subset M$.

Next we shall extend $e_{L_{S}}$ to a local section of $L$ by using the action of the complexified groups $\tilde{H}_{f}$. More precisely, set

$$
\begin{aligned}
& \tilde{\mathcal{H}}=\left\{(h, s) \in \tilde{G} \times S: h \in \tilde{H}_{\Phi(\sigma(s))}\right\}, \\
& \mathcal{H}=\left\{(h, s) \in G \times S: h \in H_{\Phi(\sigma(s))}\right\} .
\end{aligned}
$$

Then $\mathcal{H} \subseteq \tilde{\mathcal{H}}$ and the projection onto the second factor induces smooth fibrations $\tilde{r}: \tilde{\mathcal{H}} \rightarrow S$ and $r: \mathcal{H} \rightarrow S$ with $r^{-1}(s)=H_{\Phi(\sigma(s))}$ and $\tilde{r}^{-1}(s)=$ 
$\tilde{H}_{\Phi(\sigma(s))}$ for every $s \in S$. There is an obvious smooth map

$$
A: \tilde{\mathcal{H}} \longrightarrow M, \quad(h, s) \mapsto h \cdot \sigma(s)
$$

which is a local diffeomorphism along the unit section $\mathcal{E}=\{(e, s): s \in S\}$ of $\tilde{r}$. Therefore, $A$ induces a diffeomorphism of an open neighbourhood $\tilde{\mathcal{U}}$ of $\mathcal{E}$ onto an open neighbourhood $T$ of $\sigma(S)$ in $M$. Let $\mathcal{U}=\tilde{\mathcal{U}} \cap \mathcal{H}$. Then $\mathcal{U}$ maps diffeomorphically under $A$ onto an open neighbourhood $T^{\prime}=T \cap W$ of $\sigma(S)$ in $W$.

$A$ is covered by the smooth map

$$
B: \tilde{\mathcal{H}} \longrightarrow L, \quad(h, s) \mapsto h \cdot e_{L_{S}}(\sigma(s)) .
$$

Setting $\tilde{e}_{L}(A(h, s))=B(h, s)((h, s) \in \tilde{\mathcal{H}})$ we thus define a $\mathcal{C}^{\infty}$ section of $L$ over $T$.

Let $\tilde{e}_{L_{S}}^{*}$ be the dual frame of $L^{*}$ over $T$, and set $\tilde{\beta}=\left\|\tilde{e}_{L_{S}}^{*}\right\|^{2}=\left\|\tilde{e}_{L_{S}}\right\|^{-2}$. Since $G$ preserves the Hermitian structure of $L, \tilde{\beta}$ is constant along the fibres of $p_{W}: W \rightarrow W^{\sharp}$ in $T$. Then $\tilde{\beta}^{-1 / 2} \tilde{e}_{L_{S}}^{*}$ is a unitary section of $L^{*}$ over $T$, and induces a trivialization $\chi: S^{1} \times\left. T \cong X\right|_{T}$,

$$
\chi(\lambda, p)=\left(p, \frac{\lambda}{\sqrt{\tilde{\beta}(p)}} \tilde{e}_{L_{S}}^{*}(p)\right) .
$$

Suppose that $p^{\prime} \in T$ has local coordinates $(z, a+i 0)(a \in \mathbb{R})$. Pick $x^{\prime} \in \pi^{-1}\left(p^{\prime}\right)$ with coordinates $(z, a, \lambda)$. If $h \in H_{\Phi\left(p^{\prime}\right)}$ and $h \cdot p^{\prime} \in T$, then $h \cdot p^{\prime} \in T$ and it has coordinates $(z, b, \lambda)$ for some $b \in \mathbb{R}$.

Now, in view of Lemma 13 and (44) $\psi$ will parametrize $\Gamma$ if the following conditions are satisfied:

i) set $Z(\psi)=\left\{\left.(x, y) \in X\right|_{T} \times\left. X\right|_{T}: \psi(x, y)=0\right\}$; then

$$
Z(\psi)=\left\{\left.(x, h \cdot x) \in X\right|_{T} \times\left. X\right|_{T}: \pi(x) \in W, h \in H_{\Phi(\pi(x))}\right\} ;
$$

ii): $\left.d_{x} \psi\right|_{Z(\psi)}=q_{1}^{*}(\alpha),-\left.d_{y} \psi\right|_{Z(\psi)}=q_{2}^{*}(\alpha)$, where $q_{i}: X \times X \rightarrow X$ is the projection onto the $i$-th factor;

iii): passing to almost analytic extensions (and writing $\tilde{X}$ for $\left.\tilde{X}\right|_{\tilde{T}}$ ), set $\tilde{Z}(\tilde{\psi})=\{(\tilde{x}, \tilde{y}) \in \tilde{X} \times \tilde{X}: \tilde{\psi}(\tilde{x}, \tilde{y})=0\} ;$ then $\tilde{\zeta}\left(\tilde{x}, d_{\tilde{x}} \tilde{\psi}\right)=0, \tilde{\zeta}\left(\tilde{y}, d_{\tilde{y}} \tilde{\psi}\right)=$ 0 on $\tilde{Z}(\tilde{\psi})$; here $\zeta$ is the symbol of boundary $\bar{\partial}$-operator on $X, \tilde{\zeta}$ is the almost analytic extension of $\zeta$ (and, as in Theorem 4 and Lemma 13, $\Upsilon$ is defined by $\tilde{\zeta}=0$ ).

We look for a solution of the form $\phi=i(1-\lambda \bar{\mu} c(p, q))$, and view i), ii) and iii) as conditions on $c$. To determine $c$, we shall symplectically reduce $\Gamma$ with respect to the $S^{1}$-simmetry.

Namely, the action of $S^{1}$ on $X$ lifts to a Hamiltonian action on $T^{*}(X) \backslash$ $\{0\}$. Let $\nu: T^{*} X \backslash\{0\} \rightarrow \mathbb{R}$ be the moment map. These actions in turn 
almost analytically extend to actions of $\mathbb{C}^{*}$ on $\tilde{X}$ and $T^{*}(\tilde{X})$, and $\nu$ to an almost analytic map $\tilde{\nu}: T^{*}(\tilde{X}) \backslash\{0\} \rightarrow \mathbb{C}$. The symplectic reductions of $\left(T^{*}(\tilde{X}) \backslash\{0\}\right) \times\left(T^{*}(\tilde{X}) \backslash\{0\}\right)$ and $\left(T^{*}(X) \backslash\{0\}\right) \times\left(T^{*}(X) \backslash\{0\}\right)$, given by

$$
(\tilde{\nu} \times \tilde{\nu})^{-1}(1,1) / \mathbb{C}^{*} \times \mathbb{C}^{*} \supseteq(\nu \times \nu)^{-1}(1,1) / S^{1} \times S^{1}
$$

are symplectically equivalent to $T^{*}(\tilde{M} \times \tilde{M}) \backslash\{0\}$ and $T^{*}(M \times M) \backslash\{0\}$, respectively. The $S^{1}$ - and $\mathbb{C}^{*}$-invariant functions $\zeta$ and $\tilde{\zeta}$ descend to functions $\zeta_{r}$ and $\tilde{\zeta}_{r}$ on $T^{*}(M \times M) \backslash\{0\}$ and $T^{*}(\tilde{M} \times \tilde{M}) \backslash\{0\}$, and $\tilde{\zeta}_{r}$ is the almost analytic extension of $\zeta_{r}$. The reduced Lagrangian submanifold $\Gamma_{r} \subseteq T^{*}(\tilde{M} \times \tilde{M}) \backslash\{0\}$ is defined as

$$
\Gamma_{r}=:\left(\Gamma \cap(\tilde{\nu} \times \tilde{\nu})^{-1}(1,1)\right) / \mathbb{C}^{*} \times \mathbb{C}^{*} .
$$

Let $\Upsilon_{r} \subseteq T^{*}(\tilde{M}) \backslash\{0\}$ be the reduction of $\Upsilon$, that is, the almost analytic submanifold defined by $\tilde{\zeta}_{r}=0$. Given Lemma $13, \Gamma_{r}$ may be charachterized geometrically as the unique almost analytic Lagrangian submanifold of $T^{*}(\tilde{M} \times \tilde{M}) \backslash\{0\}$ contained in $\Upsilon_{r} \times \bar{\Upsilon}_{r}$ and with the given real locus, which is the reduction of the real locus of $\Gamma$.

The pull-back of $\phi$ under $\chi \times \chi$ is the function

$$
\phi^{\prime}=i(1-\lambda \bar{\mu} \gamma(p, q))
$$

on $X \times X$, where $\gamma(p, q)=c(p, q) / \sqrt{\tilde{\beta}(p)} \sqrt{\tilde{\beta}(q)}$. Hence we may view i), ii) and iii) as conditions on $\gamma$.

Over $\operatorname{diag}(W) \subseteq M \times M \subseteq \tilde{M} \times \tilde{M}$ the projection $\Gamma_{r} \rightarrow \tilde{M} \times \tilde{M}$ is a local diffeomorphism. Therefore we can find, locally along $\operatorname{diag}(W)$, a function $\tilde{\gamma}(\tilde{p}, \tilde{q})$ such that

$$
\Gamma_{r}=\left\{\left(\tilde{p}, d_{\tilde{p}} \log (\tilde{b}), \tilde{q},-d_{\tilde{q}} \log (\tilde{b})\right): \tilde{p}, \tilde{q} \in \tilde{M}\right\} .
$$

The real locus $\left(\Gamma_{r}\right)_{\mathbb{R}}$ of $\Gamma_{r}$ is the reduction under $S^{1} \times S^{1}$ of the real locus of $\Gamma$; given Lemma 10 and (44), in local coordinates we have

$$
\left(\Gamma_{r}\right)_{\mathbb{R}}=\left\{((p, \zeta), h \cdot(p,-\zeta)): p \in W, \zeta \in T_{p}^{*}(M), \exists \theta\right. \text { such that }
$$

$$
\left.\alpha_{\left(z, e^{i \theta}\right)}=d \theta+\zeta, h \in H_{\Phi(p)}\right\} \text {. }
$$

Lemma 17. If $x \in X, p=\pi(x) \in W$ and $h_{s} \in H_{\Phi(p)}$ is a smooth path, the path $\varsigma(s)=h_{s} \cdot x$ in $X$ is horizontal.

Proof. It suffices to show that if $p=\pi(x) \in W$ and $\xi \in \mathfrak{h}_{f}$, where $f=$ $\Phi(p)$, then the induced vector field $\xi_{X}$ on $X$ is horizontal at $x$, that is, $\alpha_{x}\left(\xi_{X}(x)\right)=0$. In view of (19), we are then reduced to proving that if $\xi \in \mathfrak{h}_{f}$ then $\phi_{\xi}(p)=0$. We have

$$
\phi_{\xi}(p)=\langle\Phi(p), \xi\rangle=<f, \xi>=0,
$$


in view of (43).

Lemma 18. $\tilde{\gamma}$ is constant on $W \times_{p_{W}} W$. Therefore, after adding a suitable additive constant to it, we may assume that $\tilde{\gamma}=1$ on $W \times_{p_{W}} W$, i.e. that $c(p, q)=\sqrt{\beta(p)} \sqrt{\beta(q)}$ if $p \in W$ and $q=h \cdot p$ with $h \in H_{\Phi(p)}$.

Proof. Let $\left(p_{1}, h_{1} \cdot p_{1}\right) \in W \times_{p_{W}} W$ be in the neighbourhood of $\left(p_{0}, p_{0}\right)$. Thus, $p_{1} \in W$ and $h_{1} \in H_{\Phi\left(p_{1}\right)}$. We can join $\left(p_{0}, p_{0}\right)$ and $\left(p_{1}, h_{1} \cdot p_{1}\right)$ within $W \times{ }_{p_{W}} W$ going first from $\left(p_{0}, p_{0}\right)$ to $\left(p_{1}, p_{1}\right)$ through a smooth path $v(t)=\left(p_{t}, p_{t}\right) \quad(t \in[0,1])$ with $p_{t} \in W$ for every $t \in[0,1]$, and then from $\left(p_{1}, p_{1}\right)$ to $\left(p_{1}, h_{1} \cdot p_{1}\right)$ through a path $\nu(t)=\left(p_{1}, h_{t} \cdot p_{1}\right)(t \in[0,1])$ with $h_{0}=e$ and $h_{t} \in H_{\Phi\left(p_{1}\right)}$ for every $t \in[0,1]$. It is thus sufficient to show that $\tilde{b}$ is constant on every path of the above form, and for this in turn it suffices to check that every element of $\left(\Gamma_{r}\right)_{\mathbb{R}}$ vanishes on the tangent vectors to these paths.

An element of $\left(\Gamma_{r}\right)_{\mathbb{R}}$ lying over $\left(p_{t}, p_{t}\right)$ has the form $\eta=\left(p_{t}, \zeta, p_{t},-\zeta\right)$ for a suitable cotangent vector $\zeta$ at $p_{t}$, while $\left.\frac{d v}{d t}\right|_{t}=\left(\dot{p}_{t}, \dot{p}_{t}\right)$. Thus, $\left\langle\eta,\left(\dot{p}_{t}, \dot{p}_{t}\right)\right\rangle=$ $\zeta\left(\dot{p}_{t}\right)-\zeta\left(\dot{p}_{t}\right)=0$.

An element of $\left(\Gamma_{r}\right)_{\mathbb{R}}$ lying over $\left(p_{1}, h_{s} \cdot p_{1}\right)$ has the form

$$
\eta=\left(p_{1}, \zeta, h_{s} \cdot p_{1},-\zeta_{s}\right)
$$

where $\zeta_{s}=\left(d_{p_{1}} h_{s}^{-1}\right)^{t}(\zeta)$. Here by definition $\zeta \in T_{p_{1}}^{*} M$ satisfies $\alpha_{\left(p_{1}, e^{i \theta}\right)}=$ $\zeta+d \theta$, and $d_{p_{1}} h_{s}^{-1}$ denotes the differential of $h_{s}^{-1} \in G$, viewed as a diffeomorphism of $M$, at $p_{1}$.

On the other hand, $\left.\frac{d \nu}{d t}\right|_{t=s}=(0, \dot{\kappa}(s))$, where $\kappa(s)=h_{s} \cdot p_{1}$. Thus, we need to check that $\zeta_{s}(\dot{\kappa}(s))=0$ for every $s$.

Let us consider, in the local trivialization induced by $\tilde{e}_{L}^{*}$, the path in $X$ given for some fixed $\theta \in \mathbb{R}$ by

$$
\tilde{k}(s)=h_{s} \cdot\left(p_{1}, e^{i \theta}\right)=\left(h_{s} \cdot p_{1}, e^{i \theta}\right)
$$

(the second equality reflects the construction of $\tilde{e}_{L}$ ).

By Lemma $17, \tilde{k}$ is horizontal, that is, $\alpha_{\tilde{\kappa}(s)}(\dot{\tilde{\kappa}}(s))=0$ for every $s$. Since, again invoking the definition of $\left(\Gamma_{r}\right)_{\mathbb{R}}$ and the construction of $\tilde{e}_{L}, \alpha_{\tilde{\kappa}(s)}=$ $\zeta_{s}+d \theta$, we conclude that $\zeta_{s}(\dot{\kappa}(s))=0$.

Let now $\Gamma^{\prime} \subseteq T^{*}(\tilde{X} \times \tilde{X}) \backslash\{0\}$ be the almost analytic Lagrangian submanifold parametrized by $\phi^{\prime}$. By constructon, $\Gamma^{\prime}$ satisfies conditions (ii) and (iii) above. To verify condition (i), suppose that $\left(x_{0}, y_{0}\right) \in Z\left(\phi^{\prime}\right)$ and thus $\left(x_{0}, d_{x} \phi^{\prime}\left(x_{0}, y_{0}\right), y_{0},-d_{y} \phi^{\prime}\left(x_{0}, y_{0}\right)\right) \in \Gamma_{\mathbb{R}}^{\prime}$ (the real locus of $\left.\Gamma^{\prime}\right)$. Then (in view of $[\mathbf{S Z}],(44)) d \log (\tilde{\gamma})\left(x_{0}, y_{0}\right)$ must be real. Thus, if $x_{0}, y_{0} \in X$ correspond to $\left(\left(p_{0}, \lambda_{0}\right),\left(q_{0}, \mu_{0}\right)\right)$ under $\chi \times \chi$, we must have $\left(p_{0}, q_{0}\right) \in W \times_{p_{W}} W$. Hence $\tilde{\gamma}\left(p_{0}, q_{0}\right)=1$, and the condition that $\phi^{\prime}\left(p_{0}, q_{0}\right)=1$ implies $\lambda_{0}=\mu_{0}$. Thus, by our choice of local coordinates, if $h \in H_{\Phi\left(p_{0}\right)}$ is such that $q_{0}=h \cdot p_{0}$, we 
have $y_{0}=h \cdot x_{0}$. Hence, $\Gamma^{\prime}$ satisfies (i) and the proof of Proposition 2 is complete.

Proposition 3. The regular phase function of Proposition 2 is of positive type, that is, the imaginary part of $\psi$ satisfies

$$
\Im(\psi) \geq 0
$$

on an open neighbourhood of $\left(p_{0}, p_{0}\right) \in W \times_{p_{W}} W$.

Proof. Let us go back to our original preferred holomorphic coordinates in the neighbourhood of $p_{0}$. Recall the geometric charachterization of $\Gamma$ described in Lemma 13. The condition that $\Gamma \subseteq \Upsilon \times \bar{\Upsilon}$ (Lemma 13, iii)) implies

Claim 3.2. To infinite order along $W \times_{p_{W}} W, a$ is holomorphic in $p$ and anti-holomorphic in $q$.

On the other hand, the condition that $\Gamma \supset\left(D \circ \Sigma^{\prime}\right)^{\prime}$ be the real locus of $\psi$ (Lemma 13, ii)) forces (in the given trivialization)

$$
1=\lambda \bar{\mu} a(p, q) \Longleftrightarrow((p, \lambda),(q, \mu)) \in D \circ \Sigma^{\prime}
$$

In particular, applying this to $((p, \lambda),(p, \lambda)) \in \operatorname{diag}(X)$ with $p \in W$, we obtain

$$
a(p, p)=\beta(p) \quad(p \in W) .
$$

In other words, $a(\cdot, \cdot)$ restricted to $\operatorname{diag}(W)$ is the dual hermitian metric $\beta$ on $L^{*}$, in the holomorphic coframe $e_{L}^{*}$.

In the local holomorphic coordinates $\left(z_{i}, w_{j}\right)$, with $w_{j}=a_{j}+i b_{j}, W$ is defined by the equations $b_{j}=0$. Thus, working in local coordinates near $p \in W$ and given (49), $a((z, a+i 0),(z, a+i 0))=1+|z|^{2}+|a|^{2}+\cdots$. In view of Claim 3.2 we conclude

Corollary 6. To infinite order along $W$,

$$
a\left((z, w),\left(z^{\prime}, w^{\prime}\right)\right)=1+z \cdot \overline{z^{\prime}}+w \cdot \overline{w^{\prime}}+\cdots .
$$

We can now apply verbatim the argument following the proof of Lemma 2.2 in $[\mathbf{S Z}$ ] (we only need to replace $z$ with the pair $(z, w)$ ) to conclude that $\psi$ is a positive phase function. More precisely, let us fix $x_{0} \in \pi^{-1}\left(p_{0}\right)$. We may assume that $\tilde{e}_{L}^{*}\left(p_{0}\right)=x_{0}$, so that $x_{0}$ has local coordinates $\left(z_{0}, w_{o}, \theta_{0}\right)=$ $(0,0,0)$, where $\left.\lambda=e^{i \theta}\right)$. A nearby $x \in X$ has local coordinates $(z, w, \theta)$. Let us compute $\Im\left(\psi\left(x_{0}, x\right)\right)$ as the real part of $-i \psi\left(x_{0}, x\right)$. We obtain

$$
\begin{aligned}
-i \psi\left(x_{0}, x\right) & =1-\frac{a(0,0, z, w)}{\sqrt{\beta(z, w)}} e^{-i \theta}=\left(1-e^{-i \theta}\right) \\
& +e^{-i \theta}\left[\frac{1}{2}\|(z, w)\|^{2}+O\left(\|(z, w)\|^{3}\right)\right],
\end{aligned}
$$


which is positive if $\theta$ and $(z, w)$ are small.

Given this, we may conclude:

Proposition 4. $P_{(\omega)}$ may be microlocally represented near the diagonal by an oscillatory integral of the kind:

$$
\int_{0}^{\infty} e^{i t \psi_{\omega}(x, y)} s_{\omega}(x, y, t) d t
$$

where $s_{\omega} \in S^{n}\left(X \times X \times \mathbb{R}^{+}\right)$has an asymptotic development

$$
s_{\omega}(x, y, t) \sim \sum_{j=0}^{\infty} t^{n-j} s_{\omega}^{(j)}(x, y) .
$$

By ellipticity $s_{\omega}^{(0)}(x, y) \neq 0$ if $(x, y) \in \Gamma_{\mathbb{R}}$.

With this microlocal description, to complete the proof of Theorem 3 we need now only follow the argument in the proof of Thoerem 1 on pp 327-9 of $[\mathbf{Z}]$.

Corollaries 1 and 3 are now straightforward, since the complexification $\tilde{G}$ of $G$ acts on each irreducible piece $H^{0}\left(M, L^{\otimes k}\right)_{\omega}$.

Let us now remove the simplifying assumption that $\omega$ lie in an elementary fundamental wedge for the Hamiltonian action of $G$ on $T^{*} X$. Recall that $\Psi: T^{*} X \rightarrow \mathfrak{g}^{*}$ is the moment map.

Lemma 19. Let $R \subseteq T^{*} X \backslash\{0\}$ be a $G$-invariant open conic set satisfying the following conditions:

i): the restriction $\left.\Psi\right|_{R}: R \rightarrow \mathfrak{g}^{*}$ is trasversal to $C(\mathcal{O})$;

ii): $G_{\omega}$ acts freely on $\Psi^{-1}(\omega) \cap R$.

Then $Z_{R}=\Psi^{-1}(C(\mathcal{O})) \cap R$ is a fibrating coisotropic submanifold of $R$, and the leaf of the null fibration through $y \in Z_{R}$ is the orbit of $H_{f}, f=$ $\Psi(y)$. Let $p_{Z_{R}}: Z_{R} \rightarrow Z_{R}^{\sharp}$ be the null fibration. Then the fibre product $Z_{R} \times_{p_{Z_{R}}} Z_{R} \subseteq T^{*} X \times T^{*} X$ is a Lagrangian relation. Furthermore, the orthogonal projector $Q_{(\omega)}: L^{2}(X) \rightarrow H(X)_{(\omega)}$ is microlocally equivalent on $R \times R \subseteq T^{*} X \times T^{*} X$ to a Fourier integral operator associated to $Z_{R} \times_{p_{Z_{R}}} Z_{R}$.

In other words, there is a Fourier integral operator $\tilde{Q}_{(\omega)}$ on $X$ associated to the Lagrangian relation $Z_{R} \times_{p_{Z_{R}}} Z_{R}$ such that

$$
\mathrm{WF}\left(Q_{(\omega)}-\tilde{Q}_{(\omega)}\right) \cap(R \times R)=\emptyset .
$$

Proof. This follows straightforwardly from the theory of [GS2], but we shall sketch a proof for the reader's convenience.

The first part of the Lemma is an immediate consequence of the arguments in section 2 of loc. cit.. 
On the other hand,

$$
\Gamma=\left\{(g, \gamma, \zeta, g \zeta): g \in G, \zeta \in T^{*} X \backslash\{0\}, \gamma=\Psi(\zeta)\right\}
$$

is a canonical relation in $T^{*} G \times T^{*} X \times\left(T^{*} X\right)^{-}(\operatorname{cfr}(4.2)$ of loc. cit.), called the moment Lagrangian for the action of $G$ on $T^{*} X$. In our case, this is simply the conormal bundle to the graph of the action of $G$ on $X$. Here we implicitly identify $T^{*} G$ with $G \times \mathfrak{g}^{*}$ by means of right translations, and denote by $\left(T^{*} X\right)^{-}$the symplectic manifold obtained by $T^{*} X$ by changing sign to the standard symplectic structure. Restricting to $R \times R$, we obtain a homogeneous canonical relation

$$
\Gamma_{R}=\{(g, \gamma, \zeta, g \zeta): g \in G, \zeta \in R, \gamma=\Psi(\zeta)\} \subseteq T^{*} G \times R \times R^{-} .
$$

Let furthermore

$$
\Lambda_{C(\mathcal{O})}=\left\{(g, f): f \in C(\mathcal{O}), g \in H_{f}\right\} \subseteq G \times \mathfrak{g}^{*} \cong T^{*} G
$$

be the charachter Lagrangian associated to $C(\mathcal{O})$, a homogeneous Lagrangian submanifold of $T^{*} G$. Under the hypothesis of the Lemma, $\Gamma_{R}$ and $\Lambda_{C(\mathcal{O})}$ intersect cleanly, and $Z_{R} \times_{p_{R}} Z_{R}=\Gamma_{R}^{t} \circ \Lambda_{C(\mathcal{O})}$.

Now recall that

$$
Q_{(\omega)}=\int_{G} \chi_{(\omega)}\left(g^{-1}\right) \varrho(g) d g
$$

where by $\varrho$ we denote the unitary action of $G$ on $L^{2}(X)$, and

$$
\chi_{(\omega)}=\sum_{k=1}^{\infty} \operatorname{dim}\left(V_{k \omega}\right) \chi_{k \omega} .
$$

Here $\chi_{k \omega}$ is the charachter function associated to the irreducible representation with highest weight $k \omega$. By Theorem 6.3 of [GS2], $\chi_{(\omega)}$ is a Lagrangian distribution on $G$, associated to the charachter Lagrangian $\Lambda_{C(\mathcal{O})}$; on the other hand, the Schwartz kernel $\varrho \in \mathcal{D}^{\prime}(G \times X \times X)$ of the action $\varrho$ is a Lagrangian distribution on $T^{*} G \times T^{*} X \times\left(T^{*} X\right)^{-}$, associated to the moment Lagrangian. By the above, the Schwartz kernel $\tilde{Q}_{(\omega)} \in \mathcal{D}^{\prime}(X \times X)$ of $Q_{(\omega)}$ is given by:

$$
\tilde{Q}_{(\omega)}(x, y)=\int_{G} \chi_{(\omega)}\left(g^{-1}\right) \tilde{\varrho}(g, x, y) d g .
$$

Let $P_{1}+P_{2} \sim$ id be a pseudodifferential partition of unity on $X$ such that $P_{1} \sim$ id on $R$. We may write $\tilde{\varrho}=\tilde{\varrho}_{1}+\tilde{\varrho}_{2}$, where $\tilde{\varrho}_{j}=P_{j} \circ \tilde{\varrho}$, and accordingly $\tilde{Q}_{(\omega)} \sim \tilde{Q}_{(\omega), 1}+\tilde{Q}_{(\omega), 2}$. Then $\tilde{Q}_{(\omega), 2}$ is smoothing on $R \times R$. Since on the other hand $\tilde{\varrho}_{1}$ is a Lagrangian distribution associated to $\Gamma_{R}$, by the hypothesis we may interpret $\tilde{Q}_{(\omega), 1}$ as a pull-back (under the diagonal map $G \times X \times X \rightarrow G \times G \times X \times X)$, followed by a push-forward. The Lemma then follows from the usual functorial properties of distributions. 
Let us now return to the proof of the Theorem. Given the relation between $\Psi$ and $\Phi$ on the cone $Y \subseteq T^{*}(X)$, if $\Phi$ is transversal to $C(\mathcal{O})$ then at any rate so is $\left.\Psi\right|_{Y}$, whence $\Psi$ itself in a conic open neighbourhood $R$ of $Y$ in $T^{*} X \backslash$ $\{0\}$. Given the $G$-equivariance of $\Psi$, we may assume that $R$ is $G$-invariant. By assumption, $G_{\omega}$ acts freely on $\Phi^{-1}\left(\mathbb{R}_{+} \omega\right)$, whence on $\Psi^{-1}\left(\mathbb{R}_{+} \omega\right) \cap Y$. Therefore, perhaps after replacing $R$ by a smaller $G$-invariant conic open neighbourhood of $Y$ in $T^{*} X \backslash\{0\}$, we may assume that $G_{f}$ acts freely on $\Psi^{-1}\left(\mathbb{R}_{+} \omega\right) \cap R$. Arguing as above with a pseudodifferential partition of unity, we may decompose $\tilde{Q}_{(\omega)}\left(\right.$ or $\left.Q_{(\omega)}^{\prime}\right)$ as a $\operatorname{sum} \tilde{Q}_{(\omega)}=\tilde{Q}_{(\omega), 1}+\tilde{Q}_{(\omega), 2}$, where $\tilde{Q}_{(\omega), 2}$ is smoothing on $R \times R$. Therefore, so is the composition $Q_{(\omega), 2} \circ \Pi$, since $\mathrm{WF}(\Pi) \subseteq Y \times Y \subseteq R \times R$. Every choice in the above argument may be assumed $S^{1}$-invariant. We are thus reducing to applying the previous arguments to the composition $Q_{(\omega), 1} \circ \Pi$.

\section{Proof of Proposition 1.}

The proof is a slight modification of some of the arguments in the proofs of Theorem 1 and of Theorem 2. Fix $p \in M$ with $\Phi(p) \neq 0$, and for $\epsilon>0$ let

$$
U_{p, \epsilon}=:\left\{g \in G: \operatorname{dist}_{M}(g p, p)<\epsilon\right\} .
$$

Then $U_{p, \epsilon}$ is an open neighbourhood of the stabilizer subgroup

$$
G_{p}=\{g \in G: g p=p\},
$$

and $U_{p, \epsilon} \rightarrow G_{p}$ as $\epsilon \rightarrow 0$. We have $G_{p}=\{e\}$ if $p \notin R$, since in our situation $G$ acts freely on $M \backslash R=\tilde{G} \cdot \Phi^{-1}(0)$.

Choose $x \in X$ with $p=\pi(x)$. Then $\Pi_{k, \omega}(p, p)=\Pi_{k, \omega}(x, x)$ may be decomposed as in (16) with $U=U_{p, \epsilon}$; thus the first term is the integral of an averaged Szegö kernel over $U_{p, \epsilon}$ and the second is the integral over $G \backslash U_{p, \epsilon}$. For any fixed $\epsilon$, the latter term is therefore rapidly decreasing as $k \rightarrow+\infty$, since $\Pi_{k}\left(x^{\prime}, x^{\prime \prime}\right)$ is rapidly decreasing in $k$ if $\pi\left(x^{\prime}\right) \neq \pi\left(x^{\prime \prime}\right)$. We are then led to estimate

$$
\Pi_{k, \omega}(x, x)^{\prime}=: \operatorname{dim}\left(V_{\omega}\right) \cdot \int_{U_{p, \epsilon}} \chi_{\sigma}\left(g^{-1}\right) \Pi_{k}\left(\tilde{\mu}_{g^{-1}}(x), x\right) d g,
$$

and we shall now argue that if $\epsilon$ has been chosen suitably small (in particular, very small with respect to $\varphi(p)=\|\Phi(p)\|>0)$ then $\Pi_{k, \omega}(x, x)^{\prime}$ is also rapidly decreasing as $k \rightarrow+\infty$. To this end, let us first remark that, as in (17), $\Pi_{k, \omega}(x, x)^{\prime}$ may be written as a complex oscillatory integral in $d g, d \theta, d u$, with phase $\Psi_{x}(g, \theta, u)=u \psi\left(e^{i \theta} \mu_{g^{-1}}(x), x\right)-\theta$ (here and below we think of $x$ as a parameter, and denote by $d \Psi_{x}$ the differential of $\Psi_{x}$ with respect to $(g, \theta, u))$.

Arguing as in the first part of the proof of Lemma 5, we can see that given $0<a<1<b$ there exists $d>0$ and $\epsilon^{\prime}>0$ such that if $0<\epsilon<\epsilon^{\prime}$ then $\left\|d \Psi_{x}(g, \theta, u)\right\|>d$ if $g \in U_{p, \epsilon}$ and $u \notin[a, b]$. 
Now if $g \in U_{p, \epsilon}$ we have an estimate similar to (35), with $\epsilon_{0}^{2}$ replaced by $\epsilon$, and to (37), with $C_{2} \epsilon_{0}$ replaced by $\varphi(p)$. Therefore, if $\epsilon$ has been chosen sufficiently small, the phase $\Psi_{x}$ has no stationary points on $U_{x, \epsilon} \times S^{1} \times$ $(0,+\infty)$, and actually its differential is bounded from below in norm there. This proves the first statement of the proposition.

Now let $K \subset M \backslash\left(\Phi^{-1}(0) \cup R\right)$ be a compact subset, and let us show that the rapid decay of $\Pi_{k, \omega}(x, x)^{\prime}$ is uniform over $x \in \pi^{-1}(K)$. For $\delta>0$ let $U_{\delta} \subseteq G$ be the $\delta$-neighbourhood of the identity in $G$, with respect to some fixed invariant metric. As $K$ is compact and $G$ acts freely on the semistable locus $\tilde{G} \cdot \Phi^{-1}(0) \supset K$, there exist $0<r_{K, \delta}<s_{K, \delta}$ such that

$$
\begin{gathered}
\operatorname{dist}_{X}(g x, x)<s_{K, \delta} \text { for any } x \in X \text { and } g \in U_{\delta}, \\
\operatorname{dist}_{X}(g x, x)>r_{K, \delta} \text { if } \pi(x) \in K \text { and } g \notin U_{\delta} .
\end{gathered}
$$

Furthermore, we may assume that $r_{K, \delta}<s_{K, \delta} \rightarrow 0$ as $\delta \rightarrow 0$.

Let us then choose $\delta>0$ so that $s_{K, \delta}$ is very small with respect to

$$
\varphi(K)=: \min \{\varphi(p): p \in K\}>0 .
$$

For every $x \in \pi^{-1}(K)$ we may decompose $\Pi_{k, \omega}(x, x)$ in $(16)$, where the first term is now the integral over $U_{\delta}$ and the second is the integral over $G \backslash U_{\delta}$. In view of (57), the latter term is rapidly decreasing in $k$ as $k \rightarrow+\infty$.

We are thus led to look for a uniform estimate, over $x \in \pi^{-1}(K)$, of

$$
\Pi_{k, \omega}(x, x)^{\prime}=: \operatorname{dim}\left(V_{\omega}\right) \cdot \int_{U_{\delta}} \chi_{\sigma}\left(g^{-1}\right) \Pi_{k}\left(\tilde{\mu}_{g^{-1}}(x), x\right) d g .
$$

Viewing this as a complex oscillatory integral as in (17), we want to bound from below the differential of the phase $\Psi_{x}(g, \theta, u)=u \psi\left(e^{i \theta} \mu_{g^{-1}}(x), x\right)-\theta$, uniformly over $x \in \pi^{-1}(K)$.

Given that $\pi^{-1}(K)$ is compact, a slight modification of the argument used in the proof of Lemma 5 now proves the following: If $0<a<1<b$ and $0<c<\pi$ there exists $\gamma>0$ such that for all sufficiently small $\delta>0$ we have $\left\|d \Psi_{x}\right\|>\gamma$ if $x \in \pi^{-1}(K), g \in U_{\delta}$ and $u \notin[a, b]$ or $\theta \notin[-c, c]$.

We may now argue as in the first part of the proof of this Proposition, however replacing now $\epsilon_{0}^{2}$ with $s_{K, \delta}$ in the analogue of (35), holding if $(x, g) \in$ $K \times U_{\delta}$, and $C_{2} \epsilon_{0}$ with $\varphi(K)$ in the analogue of (37).

\section{References}

[BS] L. Boutet de Monvel, J. Sjöstrand, Sur la singularité des noyaux de Bergman et de Szegö, Astérisque 34-35 (1976), 123-164

[B] M. Brion, Sur les modules de covariants, Ann. Sci. École Norm. Sup. 26 (1993), $1-21$

[BD] M. Brion, J. Dixmier, Comportément asymptotique des dimensions des covariants, Bull. Soc. Math. Fr. 119 (1991), 217-230 
[D] J. Dixmier, Les $C^{*}$-algebras et leurs réprésentations, Gauthier-Villars Paris (1964)

[GS1] V. Guillemin, S. Sternberg, Geometric quantization and multiplicities of group representations, Inven. Math. 67 (1982), 515-538

[GS2] V. Guillemin, S. Sternberg, Homogeneous quantization and multiplicities of group representations, J. Func. Anal. 47 (1982), 344-380

[GS3] V. Guillemin, S. Sternberg, Symplectic techniques in physics, Cambridge University Press 1984

[H] L. Hörmander, The analysis of linear partial differential operators I, Springer Verlag Berlin (1990)

[L] E. Lerman, Symplectic cuts, Math. Res. Letters 2 (1995), 247-258

[M] E. Meinrenken, Symplectic surgery and the Spin ${ }^{c}$-Dirac operator, Adv. Math. 134 (1998), no. 2, 240-277.

[MS] A. Melin, J. Sjöstrand, Fourier integral operators with complex valued phase functions, Springer Lecture Notes in Mathematics 459 (1975), 120-233

[S] J.-P. Serre, Complex semisimple Lie algebras, Springer Verlag New York (1987)

[SZ] B. Shiffman, S. Zelditch, Asymptotics of almost holomorphic sections of ample line bundles on symplectic manifolds, J. Reine Angew. Math. 544 (2002), 181222

[Z] S. Zelditch, Szegö kernels and a theorem of Tian, Int. Math. Res. Not. 6 (1988), 317-331

Dipartimento di Matematica e Applicazioni, Università degli Studi di Milano Bicocca, Via Bicocca degli Arcimboldi 8, 20126 Milano, Italy

E-mail address: roberto.paoletti@unimib.it 